\title{
Mechanical energy balance and apparent fracture toughness for dykes in elastoplastic host rock with large-scale yielding
}

\author{
Rémi Vachon and Christoph F. Hieronymus \\ Department of Earth Sciences, Uppsala Universitet, Villavägen 16, SE-75236 Uppsala, Sweden. E-mail: remi.e.vachon@uit.no
}

Accepted 2019 August 21. Received 2019 August 16; in original form 2019 March 21

\begin{abstract}
SUMMAR Y
The dynamics of dyke emplacement are typically modelled by assuming an elastic rheology for the host rock. However, the resulting stress field predicts significant shear failure in the region surrounding the dyke tip. Here, we model the dyking process in an elastic-perfectly plastic host rock in order to simulate distributed shear fracturing and subsequent frictional slip on the fracture surfaces. The fluid mechanical aspects of the magma are neglected as we are interested only in the fracture mechanics of the process. Magma overpressure in dykes is typically of the same order of magnitude as the yield stress of the host rock in shear, especially when the pressure effect of volatiles exsolving from the magma is taken into account. Under these conditions, the plastic deformation zone has spatial dimensions that approach the length of the dyke itself, and concepts based on linear elastic fracture mechanics (LEFM) no longer apply. As incremental plasticity is path dependent, we describe two geologically meaningful endmember cases, namely dyke propagation at constant driving pressure, and gradual inflation of a pre-existing crack. For both models, we find that plastic deformation surrounding the fracture tip enhances dyke opening, and thus increases the energy input into the system due to pressure work integrated over the fracture wall. At the same time, energy is dissipated by plastic deformation. Dissipation in the propagation model is greater by about an order of magnitude than it is in the inflation model because the propagating dyke tip leaves behind it a broad halo of deformation due to plastic bending and unbending in the relict process zone. The net effect is that plastic deformation impedes dyke growth in the propagation model, while it enhances dyke growth in the inflation model. The results show that, when the plastic failure zone is large, a single parameter such as fracture toughness is unable to capture the physics that underpin the resistance of a fracture or dyke against propagation. In these cases, plastic failure has to be modelled explicitly for the given conditions. We provide analytical approximations for the propagation forces and the maximum dyke aperture for the two endmember cases, that is, the propagating dyke and the dyke formed by inflation of a crack. Furthermore, we show that the effect of plasticity on dyke energetics, together with an overestimate of magma pressure when interpreting dyke aspect ratios using elastic host rock models, offers a possible explanation for the long-standing paradox that laboratory measurements of fracture toughness of rocks consistently indicate values about two orders of magnitude lower than those derived from dyke observations.
\end{abstract}

Key words: Fracture and flow; Plasticity, diffusion, and creep; Numerical modelling; Physics of magma and magma bodies.

\section{INTRODUCTION}

Under typical near-surface conditions, most rock types behave elastically at shear strains of less than $10^{-3}$ to $10^{-2}$ (Jaeger et al. 2009). At greater strain, rock failure occurs, either by shear fracturing in the brittle regime, or in a more ductile manner under high confining stress or under wet or hot conditions (Lawn \& Wilshaw 1975;
Ranalli 1995; Paterson \& Wong 2005). Zones of high stress are commonly encountered around fractures because their elongate geometry causes stresses to become concentrated at the fracture tips. The stress concentrations depend on the radius of curvature of the fracture tip, and it is well known that cusp-like fracture tips lead to singularities in the stress field (Lawn \& Wilshaw 1975; Tada et al. 2000). Under these conditions, inelastic effects such as plasticity or 
shear fracturing are expected to be activated in the vicinity of the fracture tip and remove the stress singularity by blunting the sharp tip (Lawn \& Wilshaw 1975; Anderson 2017).

Linear elastic fracture mechanics (LEFM) neglects the effects of inelastic deformation at the fracture tips. The advantages of using LEFM are its simplicity, the availability of analytical solutions for various boundary conditions (Sneddon \& Lowengrub 1969), and the fact that, for many engineering materials, certain observables such as the stress and strain fields are well matched by the model predictions. Moreover, LEFM leads to a simple, analytical form of the Griffith energy balance (Griffith 1921; Irwin 1957), which predicts the stability of a pre-existing fracture against propagation by comparing the surface energy required to produce new fracture surfaces to the mechanical energy released as the fracture extends. Equally rooted in LEFM is the concept of fracture toughness, which can be shown to be equivalent to Griffith's energy criterion for fracture stability. Both the energy criterion and the fracture toughness are based on an analysis of the energies and forces available for driving fracture propagation rather than the resistive mechanisms at the fracture tip. The Griffith energy balance takes the global view, while the fracture toughness results from an analysis of the stress field surrounding the fracture tip (Lawn \& Wilshaw 1975; Hertzberg 2012).

A number of fracture models have been devised which take the near-tip failure into account to varying degree while at the same time retaining as much as possible of the simplicity of the LEFM solution. These are the small-scale, the moderate-scale, and the large-scale failure models. The small-scale failure model developed by Irwin (1957) and Orowan (1955) extends the LEFM model by adding a vanishingly small process zone around the crack tip which is characterized by non-elastic behaviour. The broader stress and displacement fields are essentially unaffected so that the LEFM solution continues to be valid everywhere outside the microscopic process zone. As a result, the mechanics at the scale of the fracture decouple from the small process zone at the fracture tip, and a single parameter such as fracture toughness is sufficient to summarize the complexities of the microscopic failure process. The net effect of the small-scale failure model is an increase in the energy required for fracture propagation, and the removal of the stress singularity at the fracture tip, thus reconciling the global energy-based description of the fracture with the force balance at the tip.

Certain engineering materials such as soft steel are poorly described by the small-scale yielding model but instead have an apparent fracture toughness that varies with crack length (Anderson 2017). The size of the non-linear process zone is no longer infinitessimal, but nonetheless small in relation to the fracture length. The theory for these materials is termed moderate-scale yielding (or elastic-plastic). Formally, the process zone must be contained within the K-dominant zone (Irwin 1957), yielding an estimate for the maximum radius of the process zone of about 1/50 of the fracture length (for fracture in uniaxial tension under plain strain in an infinite medium) (Anderson 2017). For moderate-scale yielding, the critical value of the J-integral (Rice 1968) provides an alternative to the fracture toughness as a unique material property that describes the resistance against fracture propagation. Although the LEFM solution is not valid in this regime, fracture parameters such as aperture and plastic dissipation can be estimated based on analytical extensions to the LEFM model (Rice 1974; Dyskin 1997; Anderson 2017).

The final regime, large-scale yielding, comprises all systems that do not satisfy the criteria of moderate-scale yielding. There are two fundamental ways in which the moderate-scale yielding conditions may be violated. The first is if the radius of the failure zone exceeds the size of the K-dominant region (i.e. about 1/50 of the fracture length). The second relates to the derivation of the J-integral (Rice 1968), which assumes that no significant unloading occurs during fracture propagation. The literature concerning large-scale yielding conditions is rather limited because, in most engineering materials, the fracture process zone is sufficiently small so that the moderatescale yielding approximation is appropriate [a notable exception is fatigue cracking in certain types of steel (e.g. Maurel et al. 2017; Leopold \& Munier 2015)]. The process of stress unloading during fracture propagation has, thus far, attracted very little attention because most engineering studies are concerned with the stability of structures, but not with the details of the subsequent failure evolution.

In this study, we argue that in rock fracture during dyking or other hydrofracture applications, the conditions for moderate-scale yielding are commonly violated, and hence large-scale yielding must be assumed. Direct evidence of large-scale yielding in dyking is often available as observations of a failure zone that either surrounds the dyke tip, or else parallels the length of the dyke as a relict process zone. Since the length of the relict process zone, due to dyke propagation, is often identical with the length of the dyke, the relevant spatial scale of the process zone is its extent perpendicular to the dyke. As summarized by numerous authors, dykes commonly show many indications of inelastic deformation (e.g. pervasive shear fracturing, jointing, and brecciation) in a broad zone with a spatial extent that is a significant fraction of the dyke length (Delaney \& Pollard 1981; Delaney et al. 1986; Weinberger et al. 2000; Kavanagh \& Sparks 2011; Daniels et al. 2012; Abdelmalak et al. 2012; Townsend et al. 2015; Scheibert et al. 2017; Guldstrand et al. 2017). More indirect evidence of large-scale failure in rocks comes from pressure measurements during hydrofracture which indicate that more energy is lost in the fracturing process than predicted by small- or moderate-scale yielding models, and this energy is believed to be dissipated in broadly distributed inelastic deformation (Papanastasiou \& Thiercelin 1993; Papanastasiou 1997, 1999; Lecampion et al. 2018).

Since most of the fracture mechanics literature deals with yielding in tension rather than yielding due to fluid overpressure, we begin with a brief background of the Griffith energy balance, including a comparison between tensile cracking and hydrofracturing. This is followed by a summary of the concept of fracture toughness. In the main part of this contribution, we model imposed dyke propagation in a host rock with parameters typical of upper-crustal conditions. We use plasticity theory to model brittle fracturing of rock and subsequent slip on the newly created surfaces (Yu et al. 2006). In order to evaluate the stability of the dyke against propagation, the Griffith criterion is used with an additional term for plastic dissipation (Lawn \& Wilshaw 1975). The energy balance is computed for the two end member cases of inflation of a pre-existing crack and for propagation of a pressurized fracture. Analytical expressions are provided for approximate Griffith energy terms as functions of magma overpressure, host rock yield strength, and dyke length which allow rapid determination of dyke stability without numerical modeling. We then use the analytical expressions to examine the long-standing paradox that the fracture toughness of rocks as measured in the laboratory [typically in the range of $1-3 \mathrm{MPa} \mathrm{m}^{1 / 2}$ (Balme et al. 2004a; Zhang \& Zhao 2014)] is nearly two orders of magnitude lower than that computed from observations of dyke aspect ratios [50-100 $\mathrm{MPam}^{1 / 2}$ (Delaney \& Pollard 1981; Parfitt 1991; Schultz et al. 2008; Kusumoto et al. 2013)]. While the main focus of this study is on brittle rock failure, we also show results of 
the Griffith energy balance for non-linear elastic and for viscoelastic host rock rheology.

\section{GRIFFITH ENERGY BALANCE AND FRACTURE TOUGHNESS}

\subsection{Energy balance}

The Griffith energy balance is a global balance of the net energy release against the net energy uptake as a pre-existing fracture extends by an infinitesimal increment. Typically, the balance is written in terms of the macroscopic, mechanical energies on one side of the equation, which are offset by the energy uptake at the microscopic scale at the fracture tip, which is due to the formation of new free surfaces as well as inelastic dissipation in the process zone. In general, the balance may be written as

$F_{\text {tot }}=\frac{\partial W_{\mathrm{b}}}{\partial a}-\frac{\partial U_{e}}{\partial a}-\frac{\partial W_{\text {dis }}}{\partial a}$.

Here, $a$ is the half-length of the fracture or dyke, $W_{\mathrm{b}}$ is the work input at the boundaries, $U_{e}$ is the total elastic energy stored in the fracturehosting material and $W_{\text {dis }}$ is the dissipative loss due to inelastic deformations. As we will be dealing with plane fractures only, all energies here are energies per unit width in the third dimension, and the infinitesimal increase in fracture area is given by $d a$ times the unit width. The term $F_{\text {tot }}$ is the fracture extension force, which will be designated $G_{c}$ (termed the Griffith criterion) at equilibrium between fracture stability and propagation.

Most fracture mechanical literature deals with fractures in uniaxial tension rather than pressurized fractures in an unstressed medium. There exists a useful correspondence between the case of a tensile fracture in 3-D, isotropic tension and the pressurized fracture, as a constant pressure can be added to the first case to yield the solution of the latter (Timoshenko \& Goodier 1970). The correspondence between the solutions is exact as long as the rheology is independent of pressure, which is not the case for, for example Mohr-Coulomb or Drucker-Prager plasticity. The solution for the fracture in uniaxial tension is nearly identical provided that the dyke aperture is small (Timoshenko \& Goodier 1970).

Regardless of the similarities between tensile and pressurized fractures, it is instructive to examine the individual energy terms in these settings separately. For both cases, we will assume, for simplicity, that the domain of the fracture-hosting medium is infinite, so that the displacements due to the fracture and hence $W_{\mathrm{b}}$ vanish at the outer boundaries. For the tensile fracture, the fracture walls are taken to be traction-free, so that $W_{\mathrm{b}}=0$ on all boundaries (Fig. 1). Before introduction of the fracture, the material is in uniform tension so that the elastic energy density is positive and constant throughout. Introduction of the fracture results in the local release of some elastic energy $U_{e}$ in the vicinity of the fracture. The change in $U_{e}$ is negative, thus yielding a positive contribution to $F_{\text {tot }}$ in eq. (1). Inelastic dissipation always causes a reduction in the mechanical energy available for fracture propagation. How the dissipative terms are incorporated into the energy balance depends on the choice of the minimum spatial scale that is explicitly taken into account in the model. When the fracture toughness or the J-integral are used in small- and moderate-scale yielding, then the plastic deformations are not modelled explicitly, but are summarized by a single parameter. For these cases, one sets $W_{\text {dis }}=0$, and $G_{c}$ increases because the energy required to overcome the free surface energy plus the microscopic dissipation has increased. Dissipative effects below the spatial resolution are thus combined into the parameter that quantifies the resistance to fracture propagation (Anderson 2017). Similarly, every numerical model has a resolution limit which defines the representative volume element. Dissipation that is resolved by the model is then entered into the energy balance via the term $W_{\text {dis }}$, whereas the unresolved dissipation must again be added to the term $F_{\text {tot }}$. As is well known, if the large-scale dissipation depends on fracture size and geometry, then its effects cannot be summarized by a single parameter such as $G_{c}$, the fracture toughness, or the J-integral (Anderson 2017), and hence, for large-scale yielding, complete plastic models have to be run for all distinct geometries and boundary conditions. In general, a positive $W_{\text {dis }}$ reduces the mechanical energy available for propagation $F_{\text {tot }}$, thus making the fracture more stable.

Whereas all the propagation energy for the tensile fracture derives from the reduction of the initially stored elastic energy $U_{e}$, the sole source of energy for the pressurized fracture is the boundary work $W_{\mathrm{b}}$ due to the fluid pushing against the fracture walls. The initial elastic energy is zero, $U_{e}=0$, indicating that all changes $d U_{e}$ must be positive, and hence give a negative contribution to $F_{\text {tot. }}$ In other words, the stored elastic energy in the dyke model is always an energy sink. Both small-scale and large-scale inelastic deformations have the same dissipative effect as in the tensile fracture system. For a pressurized fracture in a purely elastic medium, it can be shown that the boundary work $\partial W_{\mathrm{b}} / \partial a$ is exactly balanced by the elastic energy increase $\partial U_{e} / \partial a$ if the fracture is gradually inflated from zero pressure to the final overpressure. Under these conditions, there is no excess energy available for fracture propagation. On the other hand, if the fracture is subjected to an instantaneous jump in pressure to its final value, then the boundary work is exactly doubled (see, e.g. Lawn \& Wilshaw (1975) for the equivalent argument for a tension crack that is opened either by gradual loading or by introducing an instantaneous cut in a initially loaded medium). The case with instantaneous pressure loading is most appropriate for dyke propagation as the pressure remains approximately constant during crack extension by a small increment $\mathrm{d} a$. If the applied stress on a tensile fracture is equal to the overpressure in a dyke of the same length, then the net mechanical energy available for propagation is equal in both systems. Using superscripts (t) for the configuration in tension and (p) for the pressurized dyke, the total energy release for the tensile fracture is $\partial U_{\text {tot }}^{(t)} / \partial a=-\partial U_{e}^{(t)} / \partial a$, while for the dyke that same energy is given by $\partial U_{\text {tot }}^{(p)} / \partial a=\partial W_{\mathrm{b}}^{(p)} / \partial a-\partial U_{e}^{(p)} / \partial a$. Furthermore, from the foregoing discussion it follows that $\partial W_{\mathrm{b}}^{(p)} / \partial a=-2 \partial U_{e}^{(p)} / \partial a$ and $\partial U_{e}^{(p)} / \partial a=-\partial U_{e}^{(t)} / \partial a$ (Pollard 1987; Jaeger et al. 2009).

\subsection{Fracture toughness}

The analytical solution for the stresses and displacements in an infinite elastic body containing an initially narrow slit and subjected to far-field tension was first derived by Westergaard (1933). Irwin (1957) wrote Westergaard's solution as a series expansion that is valid in the vicinity of the crack tip. The advantage of Irwin's solution is that, if only the first term of the expansion is retained, the effects of the fracture geometry and of the spatial coordinates $r$ and $\theta$ can be separated (i.e. Lawn \& Wilshaw 1975),

$\sigma=\frac{K_{I}}{(2 \pi r)^{1 / 2}} f(\theta)$,

where $\sigma$ is any of the stress components $\sigma_{x x}, \sigma_{y y}$ or $\sigma_{x y}, K_{I}$ is the stress intensity factor which depends on dyke geometry, $r$ is the 
(a)

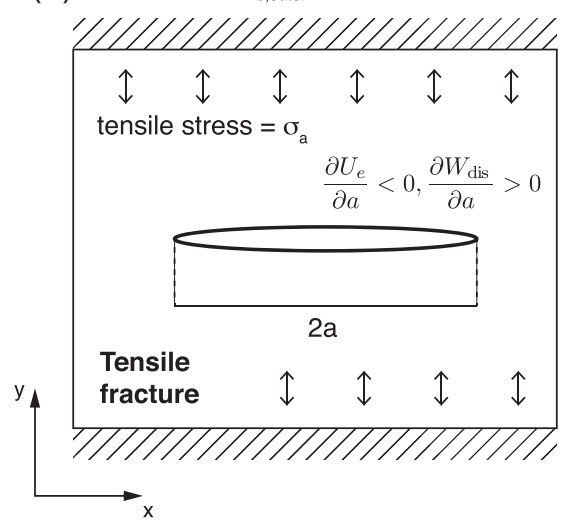

(b)

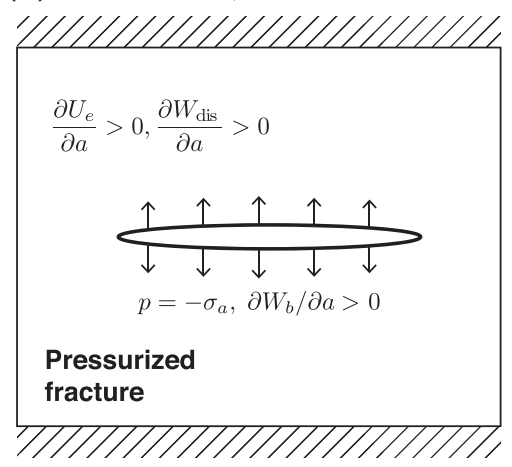

Figure 1. Comparison of energy terms for two different fracture configurations. (a) Tensile fracture. (b) Pressurized fracture or dyke. In the tensile fracture, all energy derives from the initially stored elastic energy $U_{e}$ due to applied far-field tensile stress $\sigma_{a}$. Introduction or extension of the fracture causes a drop in $U_{e}$ so that $\partial U_{e} / \partial a<0$. Dissipation by viscous or plastic flow always removes energy from the system and is thus given by $\partial W_{\text {dis }} / \partial a>0$. For the pressurized fracture, $U_{e}$ is initially zero. All energy in the system comes from the boundary work $W_{b}$ due to fluid pressure against the moving fracture wall. Some of the boundary work is expended in deforming the host material, hence the elastic deformations comprise an energy sink, $\partial U_{e} / \partial a>0$. In both fracture configurations, we assume that the work done by the distant domain boundaries is zero $\left(W_{\mathrm{b} \text {, outer }}=0\right)$.

radial distance from the dyke tip, and $f(\theta)$ describes a known dependence on angle $\theta$ which is different for each choice of stress component. The elastic region surrounding the fracture tip in which the stress field is well approximated by the lowest-order expansion given in (2) is known as the $K$-dominant region. The elastic stresses obviously approach infinity due to the cusp at the tip of the fracture. This stress singularity is avoided by introducing non-elastic deformations in the small process zone around or ahead of the fracture tip, such as the Barenblatt model which utilizes distributed cohesive stresses, or the Dugdale model which assumes local plastic deformations (Lawn \& Wilshaw 1975; Unger 2001; Hertzberg 2012).

If the non-linear process zone is much smaller than the $K$ dominant region, it is possible to define a local fracture criterion at the crack tip, namely the fracture toughness, which is equivalent to the Griffith energy criterion. The fracture toughness, denoted $K_{c}$ for opening mode (i.e. mode I) fractures is simply the stress intensity factor $K_{I}$ when fracture propagation initiates. The equivalence between the two criteria is readily demonstrated by using Irwin's stress solution (2) together with the corresponding displacement solution (Lawn \& Wilshaw 1975). The total energy due to incremental fracture propagation and opening is given by integrating $\mathrm{d} U_{c}=\sigma_{y y} u_{y} \mathrm{~d} x$ along the fracture axis over the distance of fracture extension, where $\sigma_{y y}$ is the stress perpendicular to the fracture ahead of the tip, and $u_{y}$ is the opening displacement behind the tip. Griffith's energy criterion is then recovered by taking the derivative $G_{c}=-\partial U_{c} / \partial a$. For a straight fracture in plane strain in an infinite medium, the result is $G_{c}=K_{I}^{2}\left(1-v^{2}\right) / E$, where $E$ and $v$ are Young's modulus and Poisson's ratio, respectively. A more detailed derivation is available in the literature (Lawn \& Wilshaw 1975; Unger 2001; Hertzberg 2012); the approach is outlined here merely to show that (1) the unusual units of $[P a \sqrt{m}]$ of fracture toughness are a direct result of the $r$-dependence of the only singular term in Irwin's series expansion (eq. 2) and (2) the equivalence between Griffith's criterion $G_{c}$ $=F_{\text {tot }}$ and the fracture toughness criterion $K_{c}=K_{I}$ relies critically on the assumption that the deformations surrounding the fracture are purely elastic except in a process zone which is much smaller than the $K$-dominant region.

The critical energy release rate $G_{c}$ results from a global energy balance, where each of the energy terms can be written in terms of macroscopic, measurable stresses and displacements. The fracture toughness, on the other hand, is defined in terms of the near-tip stress field. Alternatively, the fracture toughness can be computed from the observables using the appropriate stress model. However, the LEFM stress model is not appropriate for the conversion when the deformations around the fracture deviate significantly from the elastic solution. Thus, Griffith's analysis can readily be extended to fractures that are subject to broad-scale non-elastic deformations, but the fracture toughness approach becomes invalid under such conditions if the standard LEFM conversion is used.

\section{METHODS}

The objective of the present study is to analyse the Griffith energy balance of an extending dyke for different host rock rheologies. The stability of the dyke is calculated by comparing the energies of two states of the same dyke with a small difference in halflength $a$ as indicated in (1). Hence, we do not model the dynamic propagation of dykes, but only compute energies and energy differences for static dykes. Furthermore, we simplify the system by neglecting all effects of fluid flow and assume that the pressure within the dyke is constant. This condition is expected to prevail at the end of dyke propagation when the dyke has reached a state of equilibrium between propagation and stability and eventually solidifies.

\subsection{Model set-up}

The model consists of a 2-D dyke embedded in a large domain of uniform host rock in plane strain (Fig. 2). The domain boundaries are located far from the dyke, and it was verified numerically that their effect on the solution is negligible. Due to the symmetry of the set-up (Fig. 2), only one quarter of the (complete, penny-shaped) dyke and the host rock needs to be modelled. The standard set-up in fracture mechanics is that of a fracture of length $2 a$ that extends simultaneously in the positive and negative $x$-directions. However, the results may equally be interpreted in terms of a dyke of length $a$ which originates from a source at $x=0$ and extends only in the 


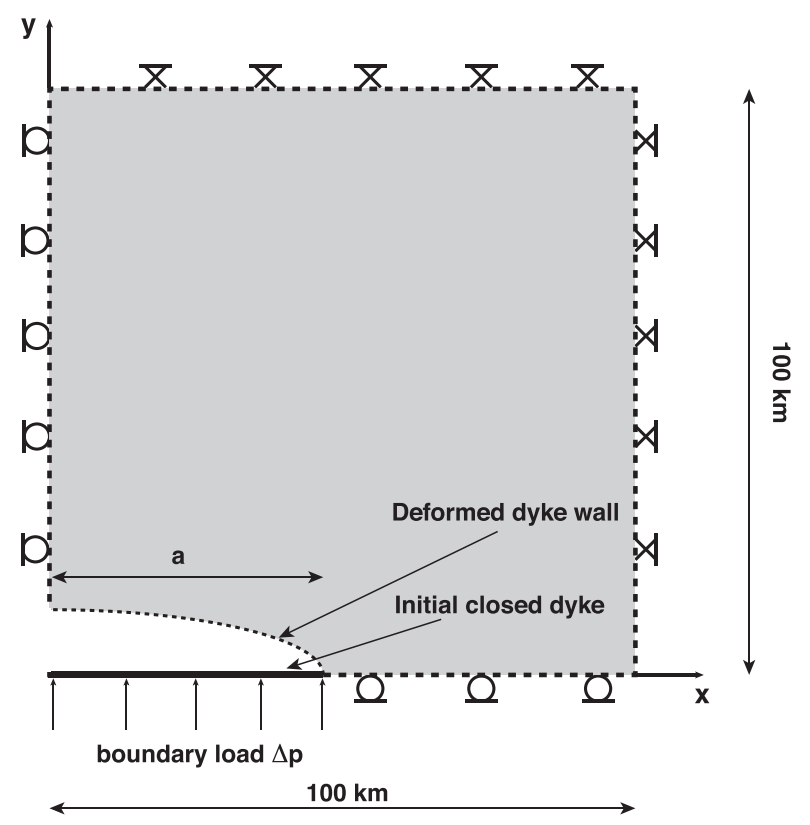

Figure 2. Model geometry and boundary conditions for all models. A closed dyke with half-length $a$ is placed inside a 2-D domain of host rock with parameters appropriate for a crustal rock (such as granite). Plane strain conditions are used in the third dimension. The domain boundaries far from the dyke are fixed and have negligible effect on the solution. The symmetry boundaries on the bottom and left of the domain are free to slip. A constant pressure is applied normal to the dyke wall.

positive $x$-direction. Since the effects of fluid flow and buoyancy within the dyke are not taken into account, the dyke itself only enters the problem as a boundary condition of constant pressure.

The domain of size $200 \mathrm{~km}$ by $200 \mathrm{~km}$ is discretized using an irregular finite-element mesh with approximately 50000 triangular elements with first-order integration and using a Lagrangian approach. The spatial resolution is greatest along the boundary parallel to the dyke plane where the element size is about $10 \mathrm{~m}$, and it decreases gradually across the domain to a size of about $10^{4} \mathrm{~m}$ at the distal boundaries. Within the host rock domain, the force balance equation is

$\nabla \cdot \sigma=0$

where $\sigma$ is the stress tensor. Inertial terms and body forces are neglected. Since the model ultimately solves the global energy balance, it is important to be aware that the quasi-static approach without inertial terms in general does not conserve energy. When inertial forces are retained, dyke inflation at constant pressure results in the excitation of inertial waves because they constitute the only mechanical mode available to take up the excess energy inherent in fracture propagation. In the quasi-static approach, the inertial waves are suppressed and the corresponding energy is lost. The energy loss is not a problem, however, because all other energy terms are computed explicitly, and from these the energy available for dyke propagation (which is equal to the energy loss) can be calculated.

For all elastic host rock models, the results are independent of the dynamic path taken to reach the final configuration. In other words, it is immaterial whether the dyke formed by slow or by instantaneous inflation, or via dyke propagation. All energy terms $U$ can thus be computed by a single dyke model with a given half length $a$ and given overpressure $\Delta p$. The propagation forces
$F$ are derivatives of $U$ with respect to $a$, and can be computed by evaluating two models with a small difference in $a$ and then calculating the derivative numerically. For incremental plasticity, on the other hand, it is well known that the results are path-dependent (Hill 1998). For a given plasticity law and for given dyke length and overpressure, there are thus infinitely many different solutions depending on how the final state was reached. We focus here on two particular paths with relevance to dyke formation. The first path, termed the 'Inflation model' (IF), describes the inflation of a pre-existing fracture. The pre-existing fracture is assumed to have formed by processes other than dyking, and it is assumed that there is no prior plastic or other inelastic deformation surrounding the fracture. The second path, termed the 'Propagation model' (PR), instead features a dyke that has formed by dyke propagation at constant magma overpressure. As in the previously described setups, dyke propagation is not modelled dynamically, but is imposed by gradually increasing length $a$ from zero to its final value. The difference relative to the IF model is that each increment in dyke length causes additional plastic strains. As in the elastic models, the propagation forces are computed by taking the numerical derivative of the energies relative to $a$, so it is always necessary to solve two models with a small difference in $a$. However, in the PR model this calculation must always be preceded by the complete imposed dyke extension beginning from $a=0$ in order to evaluate the total plastic strain before the extension step of interest.

While the dynamics of dyke propagation is not modelled, imposed dyke extension nonetheless has to be taken into account in the PR model. Depending on the numerical scheme used, this approach may require special attention in the implementation of the boundary conditions. At each location on the boundary which is initially an internal part of the host rock but, after dyke propagation, becomes part of the dyke wall, the type of boundary condition changes as a function of time. Initially, the boundary condition is of Dirichlet type (i.e. displacements are specified), but after the dyke tip has passed, the condition switches to Neumann type (stress and hence the derivative of displacement is specified). In many software packages (we use Comsol 5.3 to solve the equations), such a change in boundary conditions is not readily implemented. We use the customary approach of specifying the desired Neumann condition of constant magma overpressure along the entire boundary segment, but hold the dyke ahead of the tip closed by using spring foundations with large vertical spring constants which are connected to the boundary. The spring constants are time-dependent and are reduced to zero when the fracture tip moves past. Additionally, remeshing at every step of dyke propagation ensures that the dyke tip always coincides with a mesh node, which greatly improves the numerical accuracy of the scheme.

There is no inherent timescale in the elastic and plastic models. In the quasi-static approximation, all elastic and plastic deformations occur instantaneously, and the only timescale is that of the imposed dyke propagation, which is arbitrary. For the viscoelastic models, the timescale is discussed in the corresponding Results section.

\subsection{Rheology}

In this study, we test three different host rock rheologies: (1) linear elasticity with constant or pressure-dependent moduli, (2) elastoplasticity with constant or Drucker-Prager failure criterion and (3) viscoelasticity. The rheologies are briefly presented here. More details can be obtained from Vachon \& Hieronymus (2017). 
All models are based on isotropic linear elasticity,

$\sigma_{i j}=K \varepsilon_{k k}^{(e)} \delta_{i j}+2 G\left(\varepsilon_{i j}^{(e)}-\frac{1}{3} \varepsilon_{k k}^{(e)} \delta_{i j}\right)$,

where $\varepsilon_{i j}^{(e)}$ is the elastic strain tensor, $K$ the bulk modulus, and $G$ the shear modulus. We use the finite-strain theory for the elastic models which locally exhibit large elastic strains of several percent, while small-strain elasticity is sufficient for the other models. Unless stated otherwise, we use values of $K=50 \mathrm{GPa}$ and $G=35 \mathrm{GPa}$, typical of crystalline host rock.

For the plasticity models, we use the formulation of small-strain theory in which plastic and elastic strains are additive, that is, $\varepsilon^{(t)}=$ $\varepsilon^{(p)}+\varepsilon^{(e)}$, where $\varepsilon^{(t)}$ is the total strain, $\varepsilon^{(p)}$ is the plastic strain and $\varepsilon^{(e)}$ is the elastic strain used in (4). As a general yield criterion, we use the Drucker-Prager law

$f(\boldsymbol{\sigma})=\sigma_{\text {mises }}-C-\alpha p$,

where $C$ and $\alpha$ are constants, $\sigma_{\text {mises }}$ is the Mises stress (Hill 1998), and $p$ is the total solid pressure (i.e. the first stress invariant) in the host rock. In a first set of models, we make the yield envelope independent of pressure by setting $\alpha=0$. For these models, we use the more customary notation of $C=\sigma_{y}$, where $\sigma_{y}$ is the constant yield stress. In a second set of models, we take into account the effect of the overburden by using the complete Drucker-Prager law. In this set-up, parameter $C$ is generally termed the cohesion, and $\alpha$ the coefficient of internal friction. For these models we use values of $C=15 \mathrm{MPa}$ and $\alpha=0.25$. If fluids are present which can enter small cracks and joints and which also reduce sliding friction on fully developed cracks, then the solid pressure in (5) is replaced by $p-p_{f}$, where $p_{f}$ is the pressure of the fluid inside the pore spaces (Paterson \& Wong 2005; Albino et al. 2018). We assume that the fluid pressure and the overburden are constant throughout a given calculation, so that these effects can be separated to yield

$f(\sigma)=\sigma_{\text {mises }}-C-\alpha\left(p_{\mathrm{ob}}-p_{f}\right)-\alpha p_{\mathrm{dyn}}$,

where $p_{\mathrm{ob}}$ is the part of $p$ which is due to the constant overburden, while $p_{\text {dyn }}$ is the dynamic part of $p$ caused by the introduction of the dyke itself. The flow law in all plastic models is defined by perfect plasticity (Hill 1998).

In a second set of models, we test the effect of pressure-dependent elastic moduli in a linearly elastic material. Rocks under nearsurface conditions are typically characterized by a significant volume of open pores and cracks which reduce the elastic stiffness (Walsh 1965; Henyey \& Pomphrey 1982). As the confining pressure on these rocks is increased, the voids progressively close, and the elastic moduli tangentially approach the values of the solid rock matrix. The total variation in the elastic moduli due to pressure effects is approximately a factor of 2 in the pressure range of $0 \mathrm{~Pa}$ to about $100 \mathrm{MPa}$. For details of the empirical relations of $G(p)$ and $K(p)$, we refer the reader to Vachon \& Hieronymus (2017).

In the viscoelastic models we use the Maxwell formulation in which the total strain is given by the sum of the elastic and the viscous strains, $\varepsilon^{(t)}=\varepsilon^{(v)}+\varepsilon^{(e)}$ (Ranalli 1995; Vachon \& Hieronymus 2017). The viscous part of the rheology is given by

$\sigma_{i j}^{\prime}=2 \eta \dot{\varepsilon}_{i j}^{(v)}$

where $\dot{\varepsilon}_{i j}^{(v)}$ is the viscous strain rate, and $\eta$ is the viscosity. The tensor $\sigma_{i j}^{\prime}$ is the deviatoric part of the stress tensor, so that the viscous part of the rheology does not cause any volume changes. We do not model the thermal aspects of the dyking process as we found previously that the heat conducted from the dyke has only negligible influence on dyke opening because the heated volume of host rock is small, and because the dyke geometry makes heat transfer inefficient in the dyke tip region where it would otherwise have the greatest effect on the deformation (Vachon \& Hieronymus 2017). Instead, we use a simpler formulation with constant $\eta$, but test different values of $\eta$ to account for the background temperature of the host rock. We arbitrarily set the total time for the viscoelastic models to $1 \mathrm{yr}$ (which corresponds to the thermal diffusion timescale for a body of length scale around $5 \mathrm{~m}$ ) and test viscosities in the range of $10^{16}-10^{25} \mathrm{Pas}$. The range in viscosities is chosen a posteriori, as viscosities of $\mu>10^{25} \mathrm{Pas}$ lead to results that are identical to the purely elastic solution, while $\mu<10^{16} \mathrm{~Pa}$ s causes the dyke to become increasingly spherical in shape, so that an analysis in terms of fracture mechanics becomes inappropriate.

\subsection{Calculation of energies and propagation forces}

The energy balance of a propagating fracture comprises work done by forces on moving boundaries (in our set-up, only the dyke walls can move), the work done by dissipation due to plastic or viscous flow, and the elastically stored energy within the solid domain. The elastic energy is given by (Malvern 1969)

$U_{e}=\frac{1}{2} \int_{A} \sigma_{i j} \varepsilon_{i j}^{(e)} \mathrm{d} A$,

where the integral bounds $A$ indicate the complete elastic domain. As the model domain is $2-\mathrm{D}$ in plane strain, we compute a total elastic energy per unit length in the third dimension. Plastic and viscous dissipation are calculated using (Malvern 1969)

$\Delta W_{f}=\frac{1}{2} \int_{t} \int_{A} \sigma_{i j} \dot{\varepsilon}_{i j}^{(f)} \mathrm{d} A \mathrm{~d} t$,

where the subscript and superscript $f$ specify either the plastic or the viscous component (i.e. $W_{p}$ is plastic dissipation and $W_{v}$ is viscous dissipation). The boundary work along the fracture walls is given by (Lawn \& Wilshaw 1975)

$\Delta W_{b}=\int_{L} \Delta p \hat{n}_{i} u_{i} \mathrm{~d} L$

where $\hat{n}_{i}$ is a unit vector perpendicular to the fracture wall, $u_{i}$ is the displacement vector of the fracture wall, and the integration bounds $L$ indicate integration along the boundary of the fracture.

The Griffith energy balance (1) is given in terms of propagation forces rather than energies. For each energy term defined above, a corresponding propagation force can be computed using (Lawn \& Wilshaw 1975)

$F_{E}=\frac{\partial E}{\partial a}$,

where $E$ can be any of the energy terms $U_{e}, W_{b}, W_{p}$ or $W_{v}$.

\section{RESULTS}

In this study, we focus mainly on the elasto-plastic host rock because we believe that this rheology is most relevant for shallow dykes. For completeness, we also show results for purely elastic models with pressure-dependent elastic moduli, as well as for viscoelastic models. However, the variable elastic moduli are found to have only a relatively small effect on the dyke energetics, while viscoelasticity of the host rock requires significant heating from prior intrusions in order to affect the results significantly. 


\subsection{Linear elastic model}

We begin with a simple model with a linear elastic host rock which serves as a reference case for the subsequent models. Elastic deformations are path-independent, so there exists only a single, unique model for each final loading configuration. Fig. 3 shows the propagation forces $F_{b}, F_{e}$ and the total propagation force $F_{\text {tot }}$ for a typical crustal host rock (such as granite) with $G=35 \mathrm{GPa}$ and $K=$ $50 \mathrm{GPa}$ (corresponding to Young's modulus of $E=85 \mathrm{GPa}$ and Poisson's ratio of $v=0.22$ ), and a magma overpressure of $\Delta p=$ $10 \mathrm{MPa}$. Because of the two-dimensionality of the problem (the dyke is infinitely long in the dimension not modelled), the work done by the magma on the dyke wall as well as the elastically stored energy are both quadratic functions of dyke half-length $a$, so that the corresponding fracture propagation forces, $F=\partial U / \partial a$, are linear in $a$. There is some minor scatter of the numerical data about the best-fitting straight line due to the finite size of the elements at the fracture tip. The model reproduces well the analytical result ( $c f$. Section 2.1) that the energy input due to the boundary work, $F_{b}$, is two times the energy sink due to elastic strains, $F_{e}$, and is also equal to two times the total energy release rate $F_{\text {tot }}$. The critical energy release rate, $G_{c}$, is not calculated by our model. If fracture toughness is a material constant, then so is $G_{c}$. If $G_{c}$ is known from measurements, then our models can be used to predict the minimum fracture length at a given overpressure that will lead to dyke propagation, and is thus equivalent to LEFM fracture models based on the fracture toughness approach. Conversely, the model can be used to obtain $G_{c}$ if the dyke's length and overpressure (or length and aperture) at propagation equilibrium are known. For the linear elastic case, these relationships are known analytically $\left(F_{e}=\pi a(1-v) \Delta p /(2 G)\right.$, and $F_{\text {tot }}=-F_{e}$ ) (Sneddon \& Lowengrub 1969; Kusumoto et al. 2013), but the same concept also holds for the more complex rheologies. Typical laboratory measurements of fracture toughness of rocks are around $2 \mathrm{MPa} \mathrm{m}^{1 / 2}$, which correspond to $G_{c} \approx 60 \mathrm{~J} \mathrm{~m}^{-2}$, and which, for the given conditions, would lead to an equilibrium dyke length of less than $0.02 \mathrm{~m}$.

\subsection{Plastic host rock with constant yield stress}

As incremental plasticity is path-dependent, we show here the results for two end-member cases, which are the IF model and the PR model described in the Methods section. The most fundamental observation common to both models is that, for magma pressures of the order of the plastic yield stress, the plastic deformation zone spans an area with a characteristic length scale that is a significant proportion of the length of the dyke (Figs $4 \mathrm{a}$ and $\mathrm{b}$ ). In fact, the results shown, which are for a magma overpressure that is twice the yield strength, indicate a radius of the plastic zone that is approximately the same as the dyke's half-length $a$. A natural parameter to quantify the effect of the driving forces relative to the resistance of the rock to plastic deformation is the ratio $\Delta p / \sigma_{y}$. Due to stability problems when the plastic deformations become large in amplitude and in spatial extent, we limit our models to values of $\Delta p / \sigma_{y} \leq$ 2. On the other hand, for decreasing values of the ratio $\Delta p / \sigma_{y}$, the size of the plastic zone rapidly diminishes. From the physics of the problem, it is clear that there must always be a plastic zone present at a sharp fracture tip. However, despite the high spatial resolution of our model grid especially around the fracture tip, we find that the plastic zone is below the resolution limit when $\Delta p / \sigma_{y} \approx 0.1$, and the models are then indistinguishable from the LEFM results.

The second striking observation is the large difference between the IF model and the PR model. The IF model is characterized by plastic damage that is limited to a zone surrounding the dyke tip. This is, in fact, the type of result commonly shown for fractures in elasto-plastic media (e.g. Hertzberg 2012). In the PR model, the dyke is instead surrounded by a halo of plastic damage that extends over the entire length of the dyke. To a large degree, this plastic zone corresponds to a plastic failure zone at the dyke tip similar to that of the IF model. As the dyke propagates, this damage zone is left behind as a relict fracture tip process zone, while a new failure zone is formed around the new position of the dyke tip. Since the size of the plastic zone at the dyke tip scales with dyke length, the halo becomes increasingly broader with distance away from the dyke nucleus and toward the tip. In our PR model, dyke propagation is artificially imposed at constant $\Delta p$. Under these conditions, the broadening of the plastic halo as a function of distance is linear. In a dynamic propagation model with $\Delta p$ instead maintained at propagation equilibrium, $\Delta p$ would most likely decrease with $a$, and hence the width of the plastic halo would increase less than linearly with distance along the dyke. Simple geometric considerations indicate that the plastic halo of the PR model must contain a second stage of plastic deformation. Plasticity at the dyke tip is well known to cause a blunting of the otherwise sharp tip (Lawn \& Wilshaw 1975; Hertzberg 2012). The fracture walls in the immediate vicinity of the tip are thus plastically bent into a strongly concave shape. As the dyke tip propagates onward, it leaves behind a central portion of the dyke with nearly straight dyke walls. It follows that the material near the dyke walls behind the fracture tip has been subjected to strong bending and subsequent unbending. The total integrated plastic strain is thus significantly greater than might appear from an instantaneous picture of the final deformational state.

\subsubsection{Energy balances for the IF and the PR models}

We show results for the various energy terms for the plastic IF and PR models as functions of dyke half-length $a$, of magma overpressure $\Delta p$, and of plastic yield strength $\sigma_{y}$. Figs 5(a) and (b) show the propagation forces for both models as a function of $a$, and thus allow a direct comparison with the purely elastic result of Fig. 3 . As in the elastic model, the elastic moduli are $G=35 \mathrm{GPa}$ and $K$ $=50 \mathrm{GPa}$, and the overpressure is $\Delta p=10 \mathrm{MPa}$. The yield stress is set to $\sigma_{y}=5 \mathrm{MPa}$. For both plastic models, plastic dissipation constitutes a significant energy loss. As expected, the plastic energy loss in the PR model is greater than in the IF model because the plastic deformation zone is larger.

In addition to the relative difference in the magnitude of the plastic dissipation terms, the IF and PR models also differ greatly in the overall energy balance. In the IF model, plastic losses are slightly greater than the corresponding elastic strain energy. At the same time, there is a large increase in the boundary work relative to the purely elastic model due to increased boundary displacements. The overall effect of plasticity in the IF model is an increase in the total amount of energy available for fracture propagation relative to the linear elastic model. In other words, plasticity in the IF model enhances dyke propagation. It was found previously that plastic deformation at the fracture tip can significantly increase the total aperture of a dyke (Vachon \& Hieronymus 2017). However, the effect of this deformation on the total energy budget for dyke propagation thus far appears to have been neglected.

The situation is entirely different for the PR model. The propagation force associated with the boundary work, $F_{b}$, is similar to (but slightly greater than) that of the IF model. The plastic deformation 


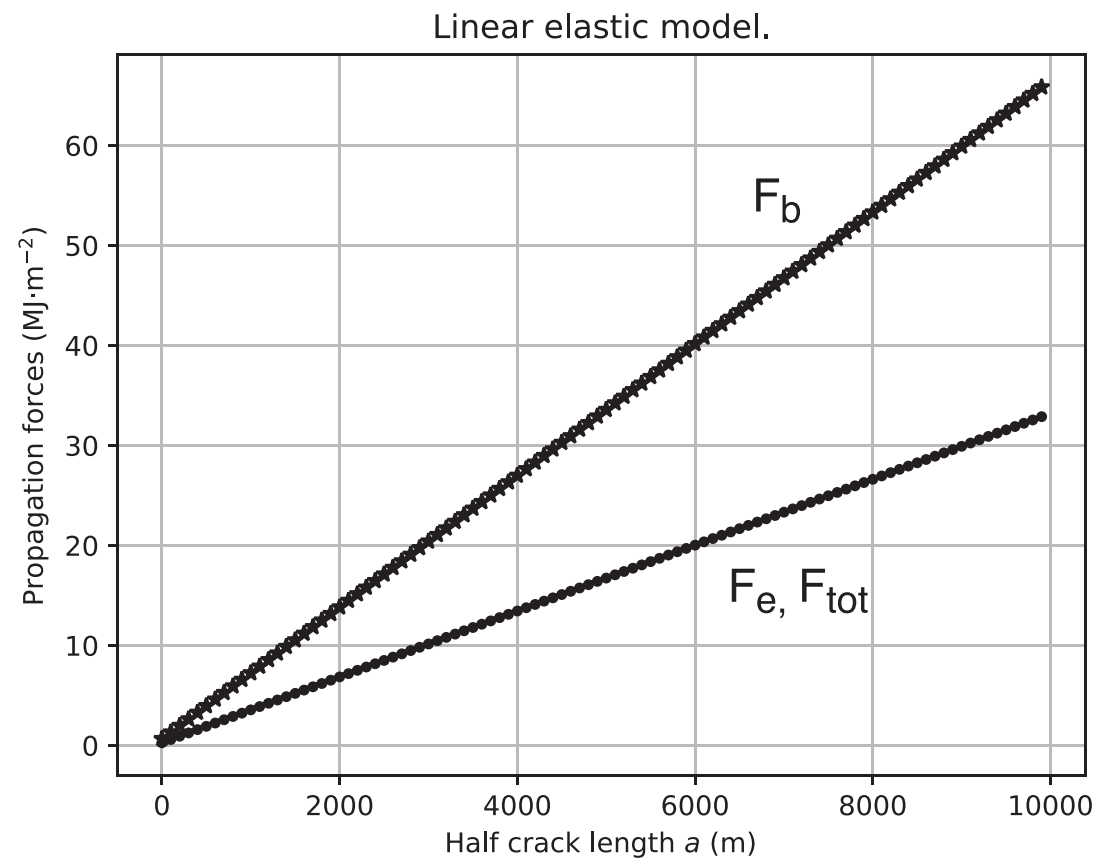

Figure 3. Propagation forces $F$ as functions of half-length $a$ for the linear elastic model with $\Delta p=10 \mathrm{MPa}, K=50 \mathrm{GPa}$ and $G=35 \mathrm{GPa}$. Dashed lines represent the best linear fit to the model data (stars and dots). $F_{e}$ and $F_{\text {tot }}$ plot on top of each other. All $F$-terms are linear in $a$ because the corresponding energies are quadratic in $a$ due to the 2-D model set-up. $F_{b}=$ force corresponding to boundary work, $F_{e}=$ force due to elastically stored energy, $F_{\text {tot }}=$ total force $=F_{b}-F_{e}$.

(a)

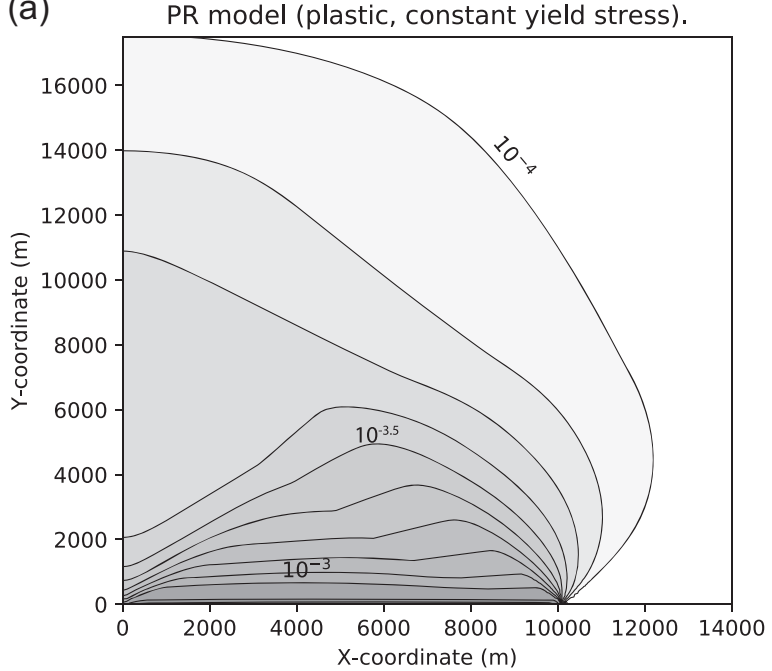

(b)

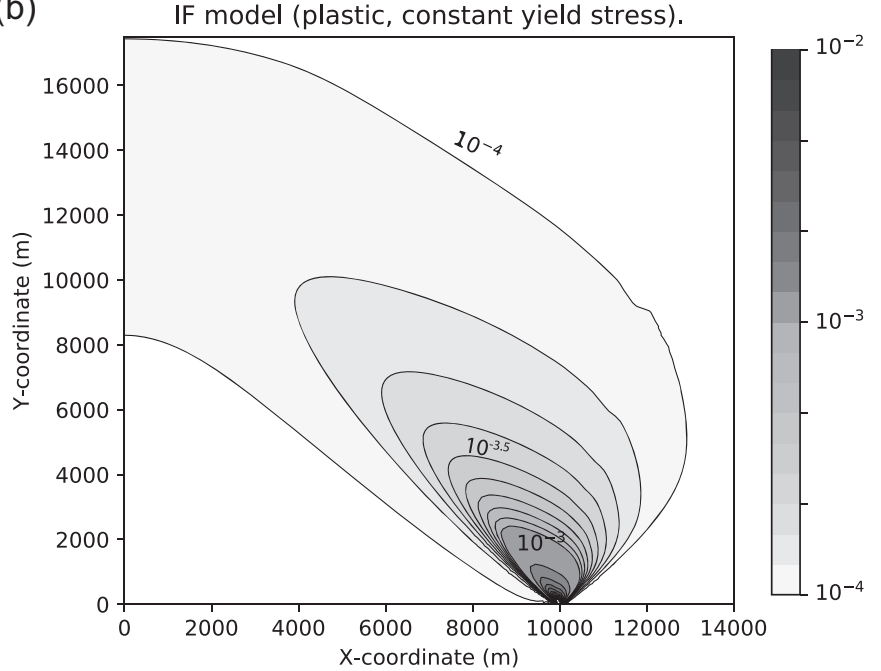

Figure 4. Plastic strain around a dyke formed by (a) propagation (PR model) and (b) inflation (IF model) in an elastoplastic host rock with constant yield stress. Result shown is for final increment when half-length $a=1 \times 10^{4} \mathrm{~m}$. Magma overpressure is set to $\Delta p=10 \mathrm{MPa}$ and yield stress $\sigma_{y}=5 \mathrm{MPa}$. For the propagation model, the dyke is surrounded by a plastic yield zone with width similar to the dyke's half-length. Much of the plastic zone is the result of the relict dyke tip when the dyke was shorter. The plastic zone may correspond to a zone of extensive jointing and brecciation sometimes observed parallel to dykes (Rubin \& Pollard 1987; Kavanagh \& Sparks 2011; Daniels et al. 2012). For the inflation model, the plastic zone surrounding the dyke tip is similar to that in the PR model, but there is no plastic damage parallel to the central part of the dyke.

surrounding the dyke tip is similar in both models, while the additional plastic losses represented by the plastic halo in the PR model do not have a significant effect on the boundary work. However, the energy loss due to plastic deformation in the PR model is so large that the total energy available for fracture propagation has dropped to near zero (Fig. 5a). In the PR model, plasticity thus makes the dykes more stable against propagation, as was often surmised in previous work to be the general case for fractures in elastoplastic media (typically expressed as an increase in fracture toughness due to plastic effects, Lawn \& Wilshaw 1975; Unger 2001; Hertzberg 2012). Since the model is scale-independent and 2-D, all energies $U$ scale as $a^{2}$, so that the corresponding propagation forces $F$ scale linearly with $a$. We therefore show best-fitting straight lines through the data. The only energy that scales differently is the free surface energy, which is usually assumed to contribute a constant loss term in a plot of $F$ versus $a$ (Lawn \& Wilshaw 1975). 
(a)

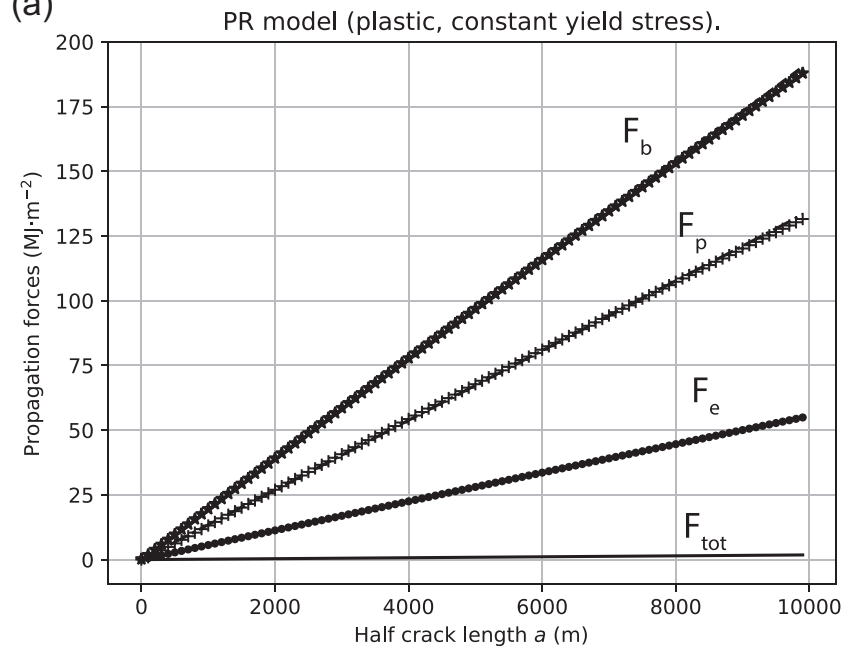

(b)

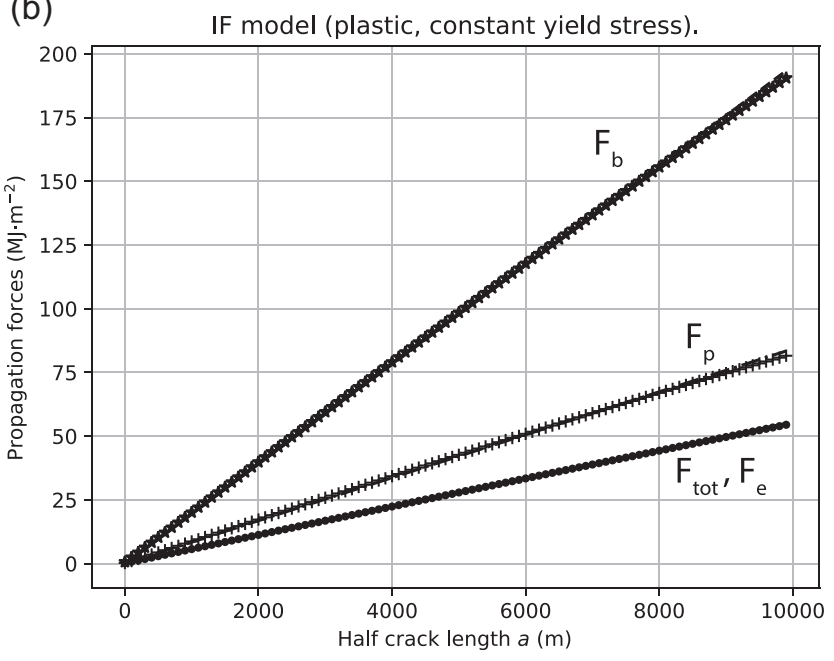

Figure 5. Propagation forces $F$ as functions of half-length $a$ for elastoplastic (a) PR model and (b) IF model with $\Delta p=10 \mathrm{MPa}, \sigma_{y}=5 \mathrm{MPa}, K=50 \mathrm{GPa}$ and $G=35 \mathrm{GPa}$. Dashed lines represent the best linear fit to the model data (stars, crosses and dots). For IF model, $F_{e}$ and $F_{\text {tot }}$ plot on top of each other. All $F$-terms are linear in $a . F_{b}$ for the IF model is similar to that of the PR model, but dissipative losses $\left(F_{p}\right)$ are reduced by a factor of about $0.5 . F_{b}=$ force due to boundary work, $F_{p}=$ force due to plastic dissipation, $F_{e}=$ force due to elastically stored energy, $F_{\text {tot }}=F_{b}-F_{p}-F_{e}$.

When the propagation forces are plotted as a function of yield stress (Figs 6a and b) or as a function of overpressure (Figs 6c and d), it is observed that the individual force terms are all strongly dependent on $\Delta p$ and $\sigma_{y}$. In the LEFM limit, $F_{b}, F_{e}, F_{p}$ and hence also their $\operatorname{sum} F_{\text {tot }}$, are all proportional to the square of overpressure, and are, of course, independent of $\sigma_{y}$. Deviations from these trends are due to the effect of plasticity. The deviations are immediately obvious in the $\sigma_{y}$-plots (Figs 6a and b), while the deviations from the $(\Delta p)^{2}$-trend are perhaps less striking (Figs $6 \mathrm{c}$ and d). However, in both cases, the effects of plasticity are large when the pressure is similar to or greater than the yield stress. For the most part, the effects of plasticity on the propagation forces can be approximated by powers of the ratio $\left(\Delta p / \sigma_{y}\right)$. The notable exception is the plastic loss term in the PR model when viewed as a function of $\sigma_{y}$. After a rapid decrease in $F_{p}$ from 5 to $10 \mathrm{MPa}$, the plastic contribution to the propagation force continues to decrease approximately linearly out to $\sigma_{y}=30 \mathrm{MPa}$. In the PR model, plasticity thus continues to have a significant effect on dyke propagation when the yield stress is at least times times greater than the magma overpressure.

The effect of plasticity on stability against dyke propagation is most clearly seen in the energy balance plots as a function of yield stress (Figs 6a and b). In the limit of high $\sigma_{y}$, the elastoplastic model is essentially elastic. For the IF model, the energy balance remains similar to the LEFM balance down to values of $\sigma_{y}$ $\approx 15 \mathrm{MPa}$ (1.5 times the magma overpressure). At $\sigma_{y}=5 \mathrm{MPa}$ ( 0.5 times the overpressure), the total propagation force $F_{\text {tot }}$ has increased to less than twice its LEFM value. The propagation force due to the boundary work, $F_{b}$ (i.e. the energy source), increases more dramatically over the same range in $\sigma_{y}$, but this increase is largely compensated by the simultaneous increase in the plastic losses $F_{p}$. Plasticity in the IF model thus makes the dyke less stable against propagation, but the effect is relatively small. For the PR model, on the other hand, plasticity decreases the net energy available for dyke propagation, and this decrease occurs over a much larger range in $\sigma_{y}$. In the LEFM limit, the total propagation force $F_{\text {tot }}$ is equal in magnitude to the propagation force due to elastic strains, $F_{e}$. At the largest value of yield stress modelled ( $\left.\sigma_{y}=30 \mathrm{MPa}\right), F_{\text {tot }} \approx$ $18 \mathrm{MJm}^{-2}$ in the PR model is about half the LEFM-limit $\left(\sigma_{y} \rightarrow \infty\right)$ of $F_{\text {tot }} \approx 33 \mathrm{MJm}^{-2}$. For decreasing $\sigma_{y}, F_{\text {tot }}$ continues to decrease more or less linearly to a value around $1-2 \mathrm{MJm}^{-2}$ at $\sigma_{y}=5 \mathrm{MPa}$. In the PR model, dyke stability thus increases significantly when plastic deformations become large.

Plastic deformation of the host rock also has a large effect on the maxiumum dyke aperture (Fig. 7). When the magma overpressure $\Delta p$ is low relative to the plastic yield stress, the increase of aperture $\delta_{y}$ is approximately linear as a function of $\Delta p$. This part of the solution is identical with the purely elastic case. Significant deviations begin to develop when the magma pressure becomes greater than the plastic yield stress. At $\Delta p / \delta_{y}=2.5$, the maximum dyke apertures in the IF model and in the PR model are greater by a factor of about 3 and 6 , respectively, than in the corresponding elastic model.

\subsubsection{Approximate analytical expressions for energies and dyke aperture in plastic models}

The strength of the LEFM models is the availability of analytical expressions for the stress and strain fields, for the energy terms, and for the displacements of the dyke walls. With these analytical expressions, it is possible to estimate, for example, the magma overpressure in dykes if the dyke length and aperture are known (Kusumoto et al. 2013). The same fundamental elastic solution also allows conversion between the critical fracture propagation force, $G_{c}$, and the fracture toughness, $K_{c}$. The analysis of the previous sections indicates that the effects of plasticity on energies and displacements are so large that they cannot reasonably be neglected. In order to allow more rapid computations without the need for solving the complete dynamic system, we provide a set of analytical approximations for the energy terms and for the dyke aperture for the IF and PR models (Table 1).

Some simple estimates of different plastic effects can be found in the literature. For example, the size of the plastic zone at the dyke tip can be estimated from the region in the elastic solution that is above the plastic yield stress (Lawn \& Wilshaw 1975; Hertzberg 2012). The total plastic dissipation can then, in principle, be computed by reducing the elastic strains in this zone at the constant yield 
(a)

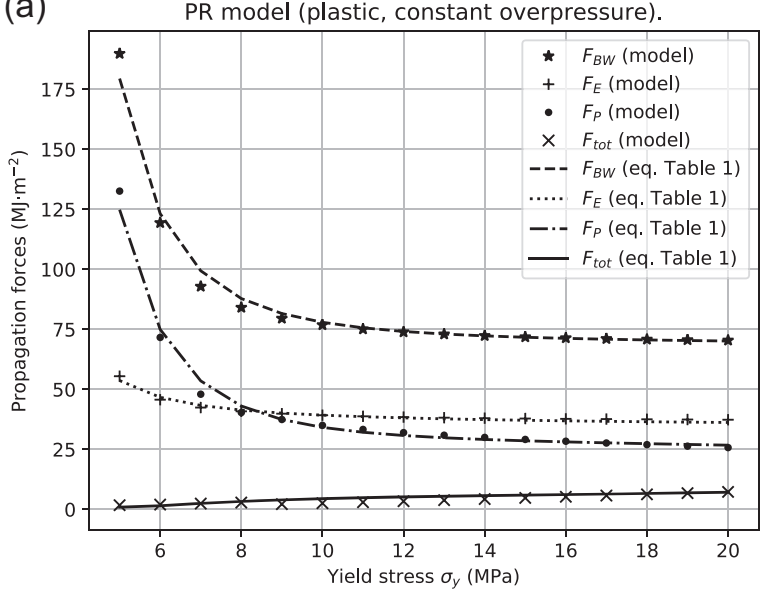

(c)

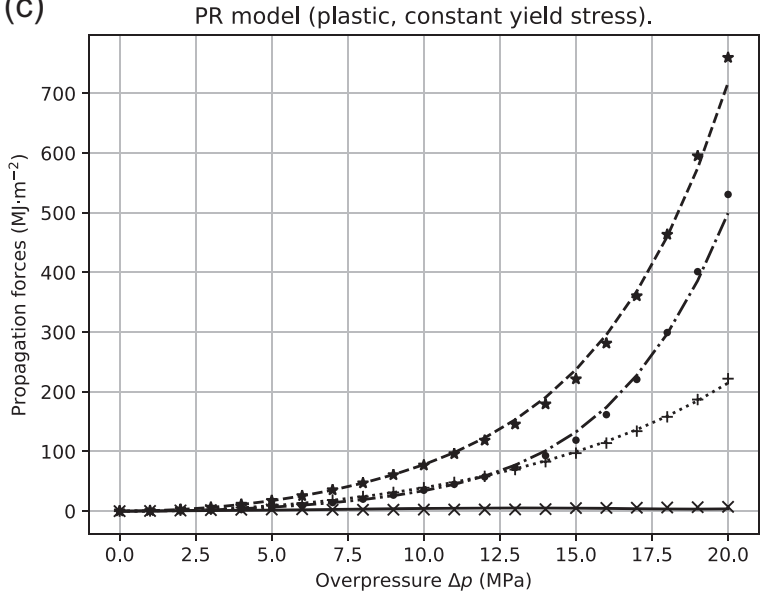

(b)

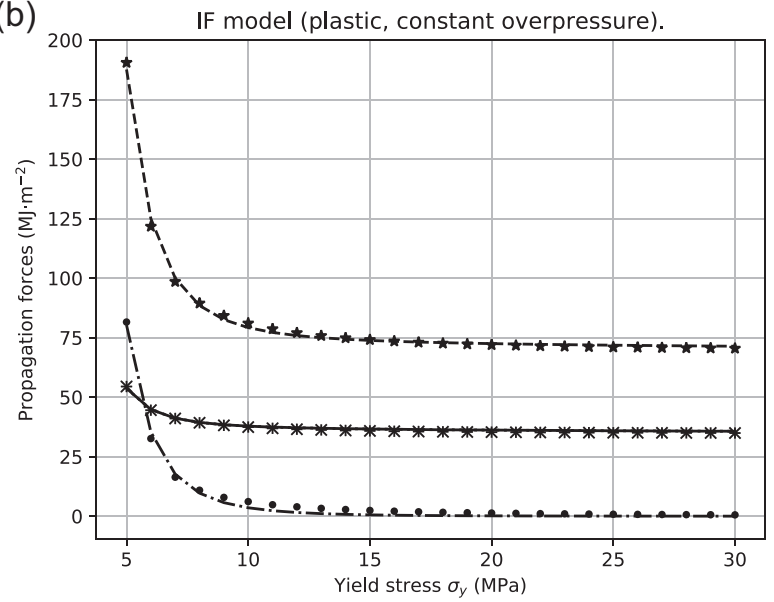

(d)

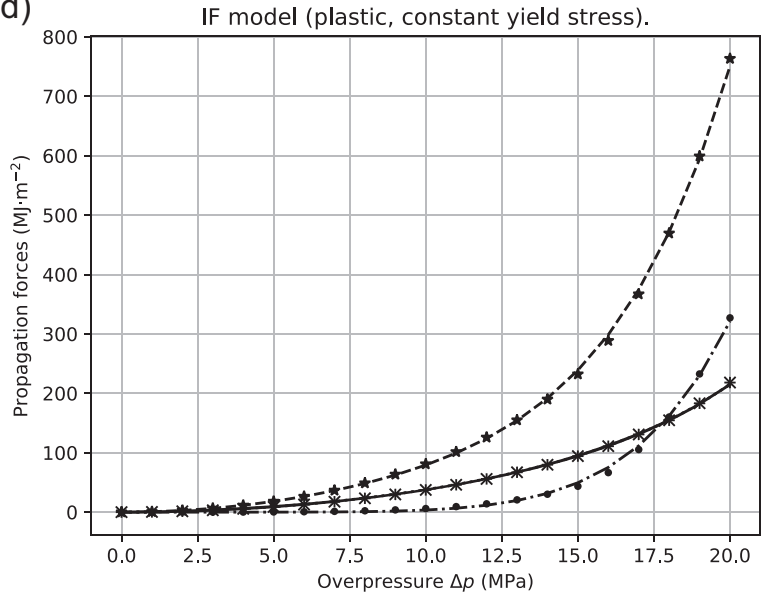

Figure 6. Propagation forces for dyke in elastoplastic host rock with pressure-independent yield stress, with $a=10000 \mathrm{~m}, K=50 \mathrm{GPa}$ and $G=35 \mathrm{GPa}$. Symbols show model results, continuous curves show analytical fit to model data (see text for details). (a, c) Dyke formed by propagation (PR model). (b, d) Dyke formed by inflation of pre-existing crack (IF model). Upper panels show forces as functions of yield stress $\sigma_{y}$ with magma overpressure set to $\Delta p=$ $10 \mathrm{MPa}$. Lower panels show forces as functions of magma overpressure $\Delta p$ with yield stress set to $\sigma_{y}=10 \mathrm{MPa}$. In the PR model, the propagation forces $F_{b}$, $F_{e}$ and $F_{p}$ increase sharply as $\sigma_{y} \rightarrow 0$, while their sum $F_{\text {tot }}$ decreases and possibly approaches zero, indicating that dykes in this configuration become more stable at low $\sigma_{y}$. Increasing $\Delta p$ in the PR model has very little effect on dyke propagation. In contrast, $F_{\text {tot }}$ in the IF model increases as $\sigma_{y} \rightarrow 0$, showing that the net effect of plasticity is to make these dykes less stable against propagation.

stress until all points in the plastic zone are at or below the failure envelope (Hertzberg 2012). It has previously been noted that the resulting approximations do not fit the plastic solutions particularly well (even in the limit of small plastic failure zones), and simple corrections are sometimes used, such as multiplying the computed dissipation by a factor of two (Hertzberg 2012). Our results confirm that estimates of the plastic solution based on the analytical elastic solution coupled with simple physical arguments generally perform poorly. We believe that this is because of the inherent non-linearity in the system, namely that plastic enhancement of dyke opening causes an increase in the size of the plastic zone and an increase in overall plastic deformation, which in turn further increases dyke opening.

We thus followed a more practical approach of using simple powers of pressure and yield stress to fit the energy curves, while largely ignoring the underlying physics. Better fits than the ones presented here can certainly be produced. However, we chose certain restrictions to our analytical functions. (1) We seek simple expressions with as few terms as possible while providing a reasonable fit. (2) The approximations must identically approach the elastic solution in the LEFM limit (i.e. when $\sigma_{y} \rightarrow \infty$ ). (3) Plasticity does not introduce any additional natural length scale, so all plastic terms must have the same dependence on dyke half-length $a$ as the corresponding elastic terms. (4) Since, in the PR model, $F_{\text {tot }}$ is typically a small value which is calculated as the sum of several much larger terms, we used the observations on $F_{\text {tot }}$ to help constrain $F_{b}, F_{p}$, and $F_{e}$.

\subsubsection{Computation of different estimates of apparent fracture toughness using the analytical approximations}

The analytical expressions of Table 1 can be used to quantify differences between different models, and to test whether the difference between the apparent fracture toughness in natural dyks and fracture toughness measured in the laboratory could be caused by the effects of large-scale yielding plasticity. The fracture toughnesses calculated from dyke aspect ratio observations are about a factor of 50-100 greater than those based on laboratory experiments (Delaney \& Pollard 1981; Parfitt 1991; Balme et al. 2004a; Schultz 


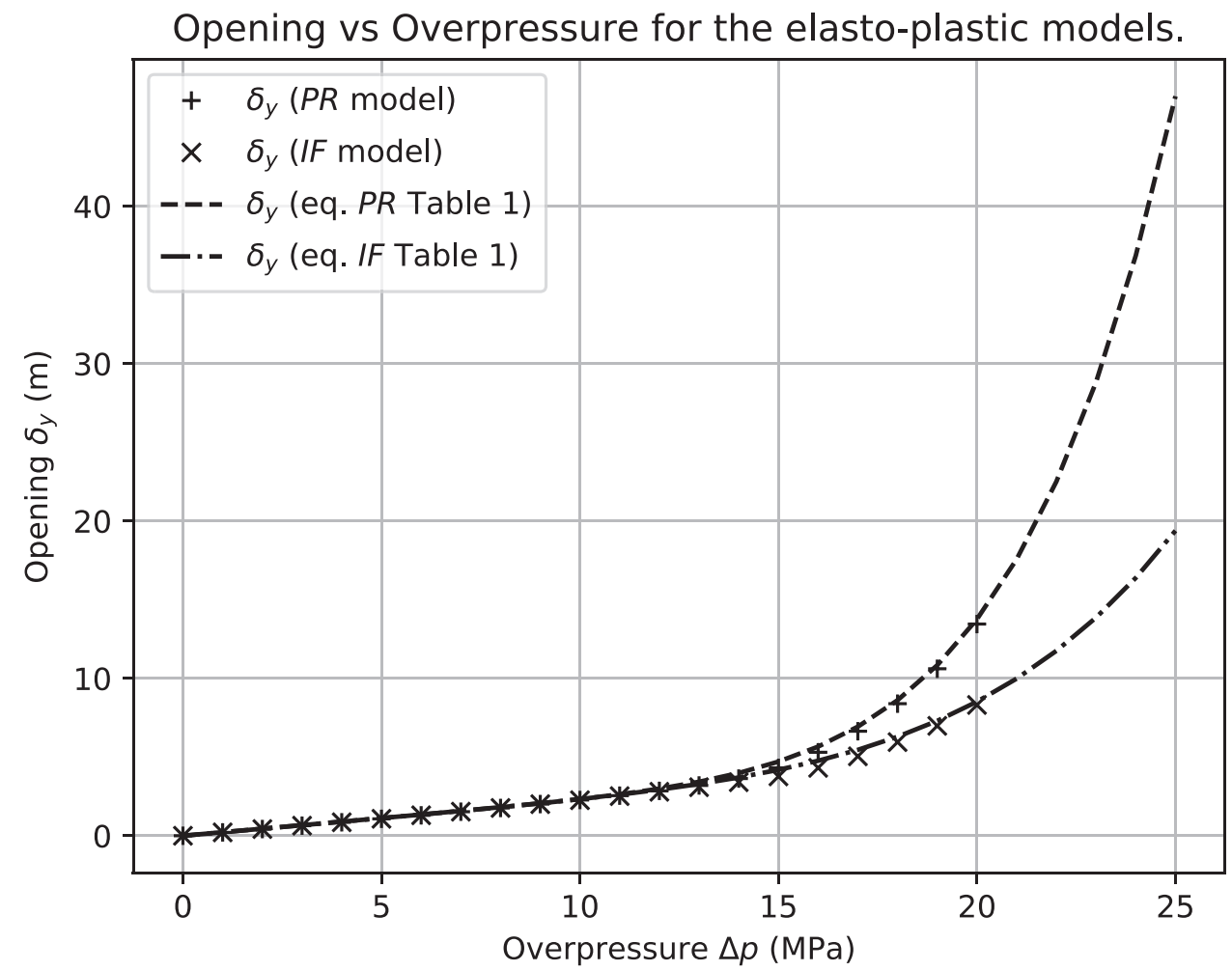

Figure 7. Maximum value of dyke aperture $\delta_{y}$ in terms of overpressure $\Delta p$ for the PR and IF elastoplastic models with constant yield stress $\sigma_{y}=10$ MPa, $a$ $=10000 \mathrm{~m}, K=50 \mathrm{GPa}$ and $G=35 \mathrm{GPa}$. The maximum aperture at the center $(x=0 \mathrm{~m})$ is greater in the PR model, but the dyke shapes differ. The IF dyke has more nearly constant thickness throughout and tapers sharply at the tip. The thickness in the PR model decreases approximately linearly from center to tip.

Table 1. Empirical expressions to fit the numerical data of the IF and PR model for plasticity with constant yield stress. Variables $U_{e}, W_{p}$ and $W_{b}$ are defined in eqs (8)-(10), $\delta_{y}$ is the maximum dyke aperture.

\begin{tabular}{ccc}
\hline Model IF & $U_{e}$ & $\frac{a^{2}(1-v)}{G}\left[\frac{\pi p^{2}}{4}+\frac{0.0817 \Delta p^{2.5}}{\sigma_{y}^{0.5}}+\frac{0.0155 \Delta p^{6.5}}{\sigma_{y}^{4.5}}\right]$ \\
& $W_{p}$ & $\frac{a^{2}(1-v)}{G} \frac{0.0828 \Delta p^{6.5}}{\sigma_{y}^{4.5}}$ \\
$W_{b}$ & $\frac{a^{2}(1-v)}{G}\left[\frac{\pi \Delta p^{2}}{2}+\frac{0.164 \Delta p^{2.5}}{\sigma_{y}^{0.5}}+\frac{0.114 \Delta p^{6.5}}{\sigma_{y}^{4.5}}\right]$ \\
$\delta_{y}$ & $\frac{\Delta p}{G}(1-v) a+\frac{\Delta p^{2} a}{32 G \sigma_{y}}\left(\frac{\Delta p}{\sigma_{y}}\right)^{3.5}$ \\
Model PR & $\left.\begin{array}{c}a_{e}(1-v) \\
G\end{array} \frac{\pi \Delta p^{2}}{4}+\frac{0.139 \Delta p^{3}}{\sigma_{y}}+\frac{0.00736 \Delta p^{6.5}}{\sigma_{y}^{4.5}}\right]$ \\
& $W_{p}$ & $\frac{a^{2}(1-v)}{G}\left[\frac{0.100 \Delta p^{6.5}}{\sigma_{y}^{4.5}}+\frac{0.746 \Delta p^{2}}{1+0.135\left(\sigma_{y} / \Delta p\right)^{2}}\right]$ \\
$W_{b}$ & $\frac{a^{2}(1-v)}{G}\left[\frac{\pi \Delta p^{2}}{2}+\frac{0.139 \Delta p^{3}}{\sigma_{y}}+\frac{0.108 \Delta p^{6.5}}{\sigma_{y}^{4.5}}-\frac{0.0216 \Delta p^{2}}{1+0.135\left(\sigma_{y} / \Delta p\right)^{2}}\right]$ \\
$\frac{\Delta p}{G}(1-v) a+\frac{\Delta p^{2} a}{32 G \sigma_{y}}\left(\frac{\Delta p}{\sigma_{y}}\right)^{4.7}$
\end{tabular}

et al. 2008; Zhang \& Zhao 2014; Kusumoto et al. 2013). The corresponding difference in critical energy release rate $G_{c}$ is a factor of 2500-10000 (since $\left.G_{c}=K_{c}^{2}\left(1-v^{2}\right) / E\right)$.

In order to determine whether this difference may be caused by large-scale plastic yielding around dykes versus small-scale yielding in the laboratory, we calculate the dyke attributes using the parametrization for the PR model (Table 1). Propagation forces $F$ are readily obtained from the energies by taking the derivatives, $F$ $=\partial U / \partial a$. The total propagation force is given by $F_{\text {tot }}=F_{\mathrm{b}}-F_{\mathrm{p}}$ $-F_{\mathrm{e}}$. An analytical expression for the total propagation force as a function of aspect ratio $\delta_{y}$ is difficult (and perhaps impossible) to obtain, but a graphical solution is straightforward by generating data vectors of $F_{\text {tot }}(\Delta p)$ and $\delta_{y}(\Delta p)$. The same quantities for the LEFM case are readily computed from the analytical expressions (Sneddon \& Lowengrub 1969). Plotting $F_{\text {tot }}$ versus aspect ratio for the elastic and for the PR plastic case (Fig. 8), where $F_{\text {tot }}=G_{c}$ at propagation equilibrium, one finds that the aspect ratios of interest are in the range of $0.003-0.005$. The corresponding magma overpressures for an aspect ratio of 0.003 are $\Delta p_{\mathrm{el}}=140 \mathrm{MPa}$ for the elastic model and $\Delta p_{\mathrm{PR}}=13.0 \mathrm{MPa}$ for the plastic PR model. At an aspect ratio of 0.005 , the pressure values are $\Delta p_{\mathrm{el}}=235 \mathrm{MPa}$ and $\Delta p_{\mathrm{PR}}=$ 13.6 MPa. The plastic yield stress in these calculations was set to $\sigma_{y}=5.0 \mathrm{MPa}$. Several points are noteworthy. First, the difference in $G_{c}$ is only partly due to plastic dissipation in the PR model. A 
PR model (plastic, constant yield stress).

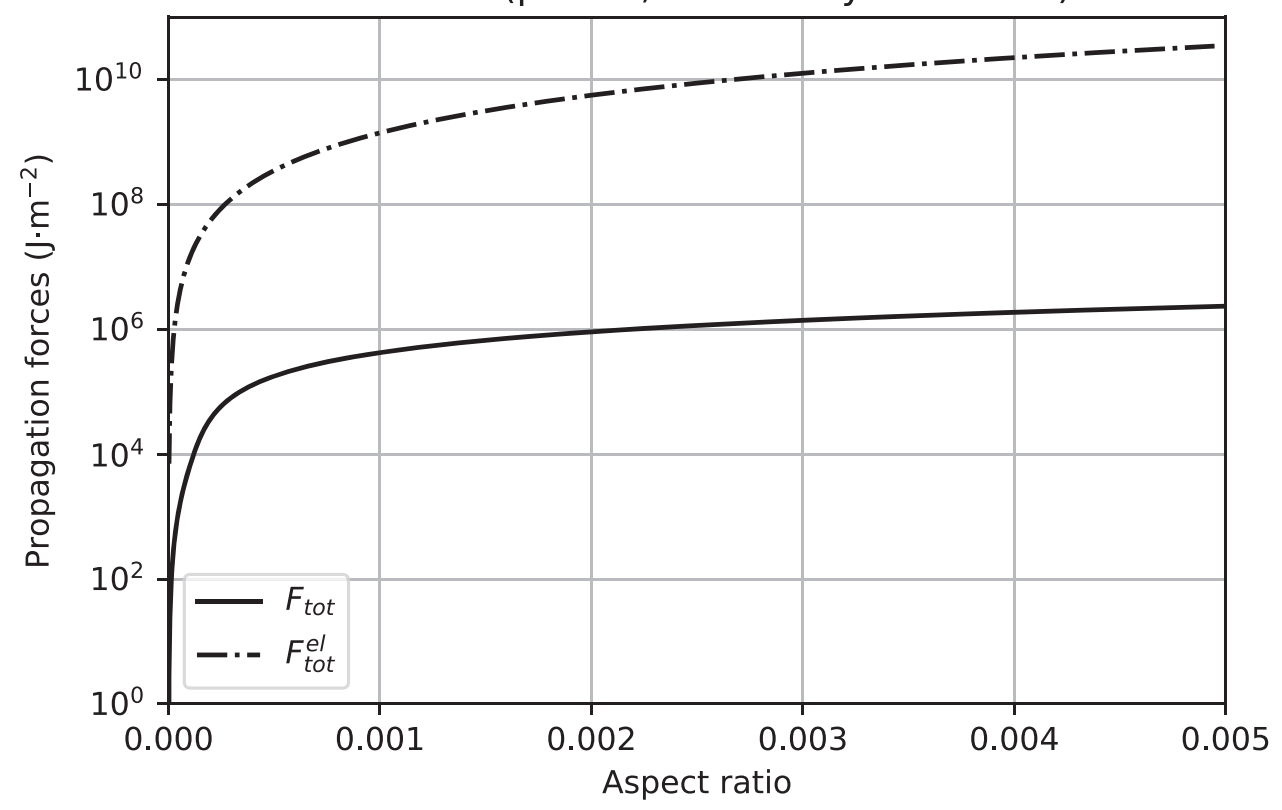

Figure 8. Total propagation forces vs. dyke aspect ratio for elastoplastic PR model with constant yield stress $\sigma_{y}=10 \mathrm{MPa}, a=10000 \mathrm{~m}, K=50 \mathrm{GPa}$ and $G$ $=35 \mathrm{GPa}$. The parameter varied is the overpressure $\Delta p$ as in Figs 6(c) and (d), but plotting $F_{\text {tot }}$ versus aspect ratio facilitates direct comparison of the critical propagation forces (since $G_{c}=F_{\text {tot }}$ at equilibrium). If the observed discrepancy in fracture toughness by a factor of 50-100 based on dyke observations versus laboratory measurements is due to plasticity of the host rock, then the $F_{\text {tot }}$ shown must differ by a factor of 2500-10 000. The corresponding aspect ratios are $0.003-0.005$. Large-scale plastic failure with a plastic zone extending about one half-length $a$ away from the dyke is required in order to provide the necessary energy dissipation (see text for details).

large proportion of the discrepancy stems from different estimates of the magma overpressure. Second, the magma overpressure in the PR model increases only by about $0.6 \mathrm{MPa}$ between aspect ratios of 0.003 and 0.005 , while the overpressure in the purely elastic model increases by $95 \mathrm{MPa}$. Finally, at around $13 \mathrm{MPa}$, the plastic PR model is well within the domain of large-scale failure. Plastic failure that is even more extensive than that shown in Fig. 4(a) is required to produce the apparent fracture toughness values that are commonly computed from dyke observations. Whereas the details of our results are dependent on the assumptions made (e.g. the exact choice of the PR model setup, or the type of plasticity), the spatial extent of the zone of plastic failure is probably quite well constrained. Plastic dissipation is required over a large volume of the host rock in order to be able to remove the required quantity of energy from the system.

The analytical expressions in Table 1 allow for a rapid check of the the effect of different parameters on the results. Varying the dyke half-length $a$ has no effect on the aspect ratio of the dyke, nor does it affect the ratio of $F_{\text {tot }}$ of the elastic model to $F_{\text {tot }}$ of the plastic model. The above analysis thus holds equally for all dyke lengths. At the same time, each of the $F_{\text {tot }}$ is proportional to $a$, so that for all models the propagation force is lower for shorter dykes. A reduction in the shear modulus $G$ results in greater dyke opening for both models, but again the ratio of the $F_{\text {tot }}$ in any one model relative to another remains unchanged.

\subsubsection{The dyke tip stress field and its relation to microscopic fracture processes}

In the small- and moderate-scale yielding models, a significant proportion of the total forces that resist fracture propagation derives from the small non-linear process zone at the tip of the fracture. The reason for this is, of course, that the effective yield stress in these models is high. The elastic solution, which stresses approaching infinity at the tip, is valid everywhere except for the small process zone in which the Mises stress is locally capped by the yield stress. In order to facilitate comparison, Fig. 9 illustrates the stress levels ahead of the fracture for the LEFM model as well as for the large-scale yielding models in the PR and the IF configuration. For all models, we show the deviatoric part of $\sigma_{y y}$, the normal stress perpendicular to the dyke plane (which is the stress component relevant to mode I fracturing), as a function of $r^{-1 / 2}$. The yield stress for both plastic models is set to $\sigma_{y}=5 \mathrm{MPa}$. The stresses in the plastic models are relatively similar to the elastic solution, albeit slightly higher, in the far field and up to the location where $\sigma_{y y}$ approximately reaches the yield stress $\sigma_{y}$ (note that the yield stress sets a limit for the Mises stress, but not to individual stress components such as $\sigma_{y y}$ ). From this point and up to the fracture tip (at $1 / \sqrt{r} \rightarrow \infty$ ), the stress solution is more or less constant and remains close to the yield stress.

With the observed near-tip stress field (Fig. 9) constant down to the smallest scale resolved (due to the assumption of perfect plasticity), there is no reason from the physics of the system to expect different behaviour at the smaller, unresolved scales. This is an important result for the determination of any fracture toughness that might have to be added to the macroscopic large-scale yielding models due to processes that are unresolvable for a given numerical scheme. The reason that the inelastic deformations in the vanishingly small process zone of the small-scale yielding models contribute significantly to the fracture toughness is because the elastic stresses reach extremely large values at the boundary of the process zone, and the stresses inside the process zone are thus correspondingly large. The large-scale failure model is instead characterized by constant and relatively low stress levels. The contribution of the 


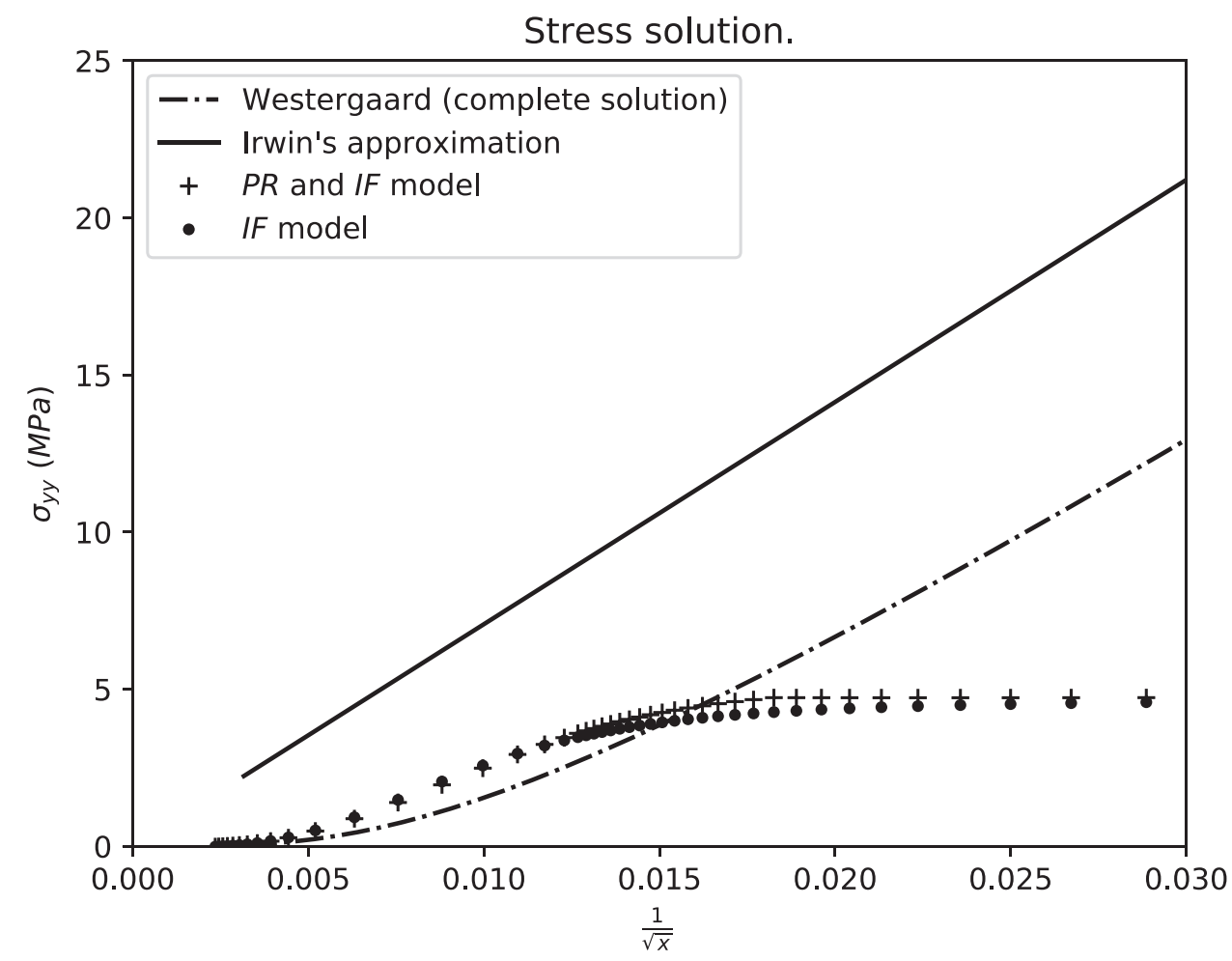

Figure 9. Normal stress $\sigma_{y y}$ ahead of the dyke tip for elastic and plastic models with yield stress $\sigma_{y}=5 \mathrm{MPa}$. Stress is plotted as a function of $1 / \sqrt{x}$ for better comparison of the plastic solution with the analytical elastic solution close to the tip located at $x=0$. Near the dyke tip, stresses in the plastic models are characterized by a stress plateau close to the value of $\sigma_{y}$.

microscopic failure zone at the dyke tip is thus negligible due to its infinitesimal size combined with the moderate stress level which is close to $\sigma_{y}$, provided that the assumption of constant yield stress extends to the smalles scales at the fracture tip.

\subsubsection{Plasticity with pressure-dependent failure}

Rock fracture is generally dependent on the solid pressure due to overburden, and on fluid pressure if there are fluids present that can aid in opening new cracks. This type of rock behaviour can be modelled with the Mohr-Coulomb failure envelope, or with the closely related but numerically more stable Drucker-Prager model which we use here. Critical to the model results is how the fluid pressure $p_{f}$ affects plastic yielding. If the only fluid available is the magma, then there is probably little or no fluid pressure effect on the effective yield stress because most magmas are relatively viscous and will not be able to penetrate into small cracks or joints (i.e. $p_{f}=$ 0 in eq. 6). This consideration is also supported by the evidence, as dykes or dyke tips are generally not surrounded by a magma-filled fracture network. On the other hand, volatiles which exsolve from the magma have much lower viscosities and are thus able to enter even very small cracks. The volatile pressure is probably not constant, but depends on the volume of volatiles available versus the volume of cracks and voids which are to be filled by the volatiles. Furthermore, the volatile pressure is probably a function of the distance from the magma source. We neglect these complications and assume a constant volatile pressure. We consider two end-member models, one with zero volatile pressure, and one with the volatile pressure equal to the magma pressure. We restrict our analysis to the PR model set-up, and we set the friction coefficient to $\alpha=0.25$ and the cohesion to $C=15 \mathrm{MPa}$.
When the fluid pressure in the Drucker-Prager formulation is set to zero, the model results are similar to those with a constant yield stress, except that the total yield stress is now equal to the cohesion $C$ plus $\alpha$ times the effect of the overburden $p_{\mathrm{ob}}$ (see eq. 6). The effects of the dynamic pressure (i.e. the non-deviatoric stress in the solid domain) on the energy balance are observed to be minor (Fig. S1). At depth, the overburden increasingly impedes plastic deformations. At the maximum value of overburden pressure tested $\left(p_{\mathrm{ob}}=200 \mathrm{MPa}\right.$, corresponding to a depth of about $8 \mathrm{~km}$ ), the effect of plasticity on the energy balance is significantly diminished. The plastic propagation force $F_{p}$ is reduced by a factor of about 10 relative to its maximum value, but with a magnitude of about 0.1 times the total propagation force $F_{\text {tot }}$, it is not negligible (Fig. S1).

In the second set of models, the fluid pressure in the yield stress is set equal to the magma pressure. The fluid pressure completely cancels the effect of the lithostatic overburden, and further reduces the cohesion by an amount of $\alpha$ times the magma overpressure (i.e. the magma pressure above the lithostatic value, see eq. 6). The model results are similar to the corresponding models with constant yield stress $\sigma_{y}$, with the dynamic pressure again only causing minor modifications (Fig. S2).

\subsection{Pressure-dependent elastic moduli}

For the elastic model with pressure-dependent moduli, the results (Fig. S3) are similar to the LEFM solution (Fig. 3) as there are no dissipative processes present that affect the energy balance. There is a small difference between the two models as the propagation forces due to boundary work $F_{b}$ and due to elastic strain, $F_{e}$, are higher (by around 30 per cent) for the pressure-dependent model. This increase in energies results from the greater opening of the dyke due to the 
reduced elastic moduli in the region of tension or low pressure near the dyke tips. As a result, dykes from the pressure-dependent elastic model are thicker and have a more pronounced taper near the ends than the elliptical dykes from the linear elastic solution (Vachon $\&$ Hieronymus 2017). The reduction of the elastic moduli at low pressure also results in an increase in the net energy available for propagation relative to the LEFM model, indicating that fracture propagation is enhanced by the given type of pressure-dependence.

\subsection{Viscoelasticity}

The dynamics of a dyke intruding into a viscoelastic host rock is more complex than the elastic and plastic cases because it introduces two additional timescales, namely that of heat diffusion from the dyke, and that of the viscous flow law. Together with the timescale of dyke propagation, this leads to three independent timescales in the problem. The purpose of the present study is not to provide an exhaustive parameter study of the viscoelastic problem, but rather to show similarities and differences relative to the elastic and plastic cases, and to give some indication of how viscous deformation of the host rock may affect dyke propagation.

In order to do so, we make the following simplifying assumptions. Intrusion of the dyke is assumed to occur instantaneously, thus removing the timescale of dyke propagation. Diffusion of heat from the dyke into the surrounding host rock is largely limited to the dyke walls (rather than the dyke tip) and is thus ineffective in causing significant shear flow by reducing the viscosity (Vachon \& Hieronymus 2017). We therefore neglect heat diffusion altogether (thus removing the corresponding timescale from the problem), and focus instead on the background viscosity of the host rock, which may vary due to rock type, temperature and pressure. We show results for the viscosity range $\mu=10^{16} \mathrm{~Pa}$ s (viscosity of wet quartzite near the melting point, (Hirth et al. 2001)) to $\mu=10^{22} \mathrm{~Pa} \mathrm{~s}$ (viscosity above which viscous deformations become negligible over the timescales of dyke cooling).

As the model evolves in time due to viscous flow, we set a final time of $t_{\text {final }}=1 \mathrm{yr}$ at which we terminate the model. In nature, dyke inflation stops when the dyke has solidified. Our choice for $t_{\text {final }}$ corresponds to the timescale of thermal diffusion for a dyke of thickness $30 \mathrm{~m}$. The half-length of the dyke is set to $a=10^{4} \mathrm{~m}$, and the elastic parameters are as in the LEFM model.

The most important result is that, for the given model parameters, the viscous deformations can be neglected for viscosities of about $\mu=10^{19}$ Pa s or greater (Fig. 10). For greater viscosities, viscous dissipation becomes negligible and the other energy terms approach the values observed for the LEFM case (Fig. 3, at $a=10^{4}$ ). The viscoelastic Maxwell time may be defined as $\tau=\mu / G$, which yields $\tau=2.9 \times 10^{8} \mathrm{~s}$ (for $\mu=10^{19} \mathrm{Pas}$ and $G=35 \mathrm{GPa}$ ). Comparing with the model timescale $t_{\text {final }}$ of 1 yr $\left(\approx 3 \times 10^{7} \mathrm{~s}\right)$, we conclude that viscous effects can be neglected over timescales up to one tenth of the Maxwell time.

Over longer timescales, viscous flow causes deformations that perturb and eventually dominate the mechanical energy balance of the dyke. At $t_{\text {final }}=\tau$ (i.e. $\mu=10^{18} \mathrm{~Pa}$ s in Fig. 10), the propagation force due to boundary work, $F_{b}$, has increased from the LEFM value of $65-110 \mathrm{MJ} \mathrm{m}^{-2}$. The propagation force due to viscous dissipation, $F_{\mathrm{v}}$, increases by the same amount, from 0 to $45 \mathrm{MJ} \mathrm{m}^{-2}$, so that the total propagation force $F_{\text {tot }}$ remains essentially unchanged. Both $F_{b}$ and $F_{v}$ continue to increase with decreasing viscosity, while $F_{\text {tot }}$ remains constant to just below $\mu=10^{-17} \mathrm{Pas}$, which corresponds to just over 10 times the Maxwell time. Below this value of $\mu$, the propagation force due to elastic deformations suddenly increases sharply. At this point, the magma intrusion turns from an initial shape of low aspect ratio toward a shape with aspect ratio of unity (i.e. it becomes increasingly similar to a circle). We conclude that the present analysis, which is based on concepts of fracture mechanics, is valid for viscoelastic host rock if the timescale is of the order of 10 Maxwell times or less.

\section{DISCUSSION}

Host rock rheology has a large effect on the stability of dykes against propagation. For dissipative rheologies such as viscoelasticity and viscoplasticity, there are two effects which tend to counteract each other. The more obvious effect is that energy is dissipated which would otherwise have been available for dyke propagation. The second effect is that the dyke aperture increases and hence causes an increase in the work done by the magma pressure on the dyke walls. For dykes in an elastoplastic host rock, the history of the dyke's evolution plays a critical role. For dykes that form by inflation of a pre-existing crack, the additional work input by the boundary forces dominates over the dissipative effects, thus making the dyke more prone to propagation. For dykes that form by propagation at constant magma pressure, the total energy dissipation is larger and able to overcome the effects of the boundary work, thus making the dyke more stable against propagation. While in this study we focus on the mechanics of dyke propagation, the results hold equally for tensile fractures. In the absence of a fluid that fills the fracture, there is no effect of boundary work. However, the elastic energy stored initially will in this case be reduced by a corresponding amount due to the plastic deformations at the fracture tip. With the large-scale numerical model, we are not able to test whether the interactions between dissipation and boundary work extend to models with infinitesimally small plastic zones. However, it seems likely that plasticity in a microscopically small non-linear zone at the fracture tip will result in a small increase in dyke aperture, and hence cause an increase in the boundary work $F_{b}$. For small-scale plasticity to have a stabilizing effect on fracture propagation, the effect of plastic dissipation must dominate over the $F_{b}$-term. In the large-scale plasticity models, we show that this is the case in the PR models, but not in the IF models.

In all plastic models, we find that the energy terms of the Griffith balance are strongly perturbed from their LEFM values if the magma overpressure is similar to or greater than the plastic yield stress. The question is how often these conditions are fulfilled. Magma overpressure in dykes is generally believed to be in the range of a few MPa to a few tens of MPa (Pollard \& Muller 1976; Poland et al. 2008; Geshi et al. 2010; Daniels et al. 2012; Kusumoto et al. 2013), although much higher overpressures of up to several hundreds of MPa have been reported (Poland et al. 2008; Daniels et al. 2012). Much of the information on pressures in magmatic plumbing systems derives from measurements of dyke aspect ratios, which are then converted to magma pressures using the LEFM solution for dykes in elastic host rock. In particular, the highest overpressure values that have been computed are probably overestimates because an incorrect rheology has been assumed (Vachon \& Hieronymus 2017). Plastic deformation in our models is used as a numerical analogue of brittle fracture and frictional sliding. Both mechanisms are strongly affected by the lithostatic overburden and by fluid pressure. Without the aid of fluid pressure, the effect of overburden suppresses extensive brittle shear fracturing around the dyke tip unless the dyke is very shallow. However, if the fluid pressure due to 
IF model (Viscoelastic model).

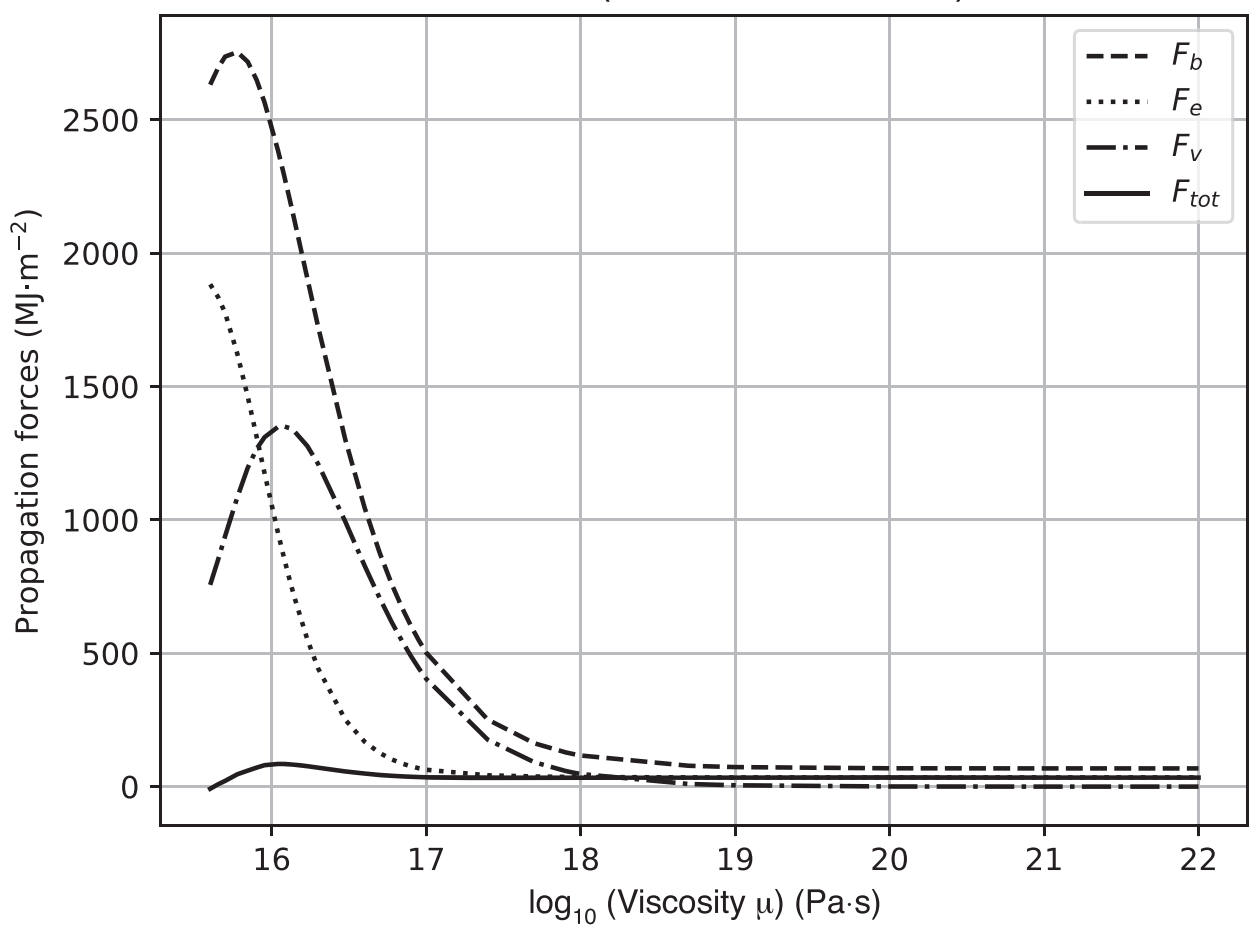

Figure 10. Propagation forces $F$ as functions of viscosity $\mu$ for the viscoelastic IF model with $\Delta p=10 \mathrm{MPa}, a=10000 \mathrm{~m}, t=1 \mathrm{yr} K=50 \mathrm{GPa}$ and $G=$ $35 \mathrm{GPa}$. Viscous dissipation has a significant effect on the energy balance for viscosity values around $\mu=10^{18}$ Pas or lower. When the viscosity is high $\mu>$ $10^{19} \mathrm{~Pa}$ s the model is similar to the elastic solution. For the lowest viscosities $\left(\mu<10^{16} \mathrm{~Pa}\right.$ ), the fracture approaches a circular shape over the chosen model time of $1 \mathrm{yr}$. The analysis in terms fracture propagation thus loses its validity at very low host rock viscosities.

volatiles exsolving from the magma is of the same order as the total magma pressure, then the effect of the overburden will be approximately cancelled. The shear stresses then only need to overcome the cohesion minus the remaining effect of the magma overpressure. Typical values of rock cohesion range from about $10 \mathrm{MPa}$ for poorly consolidated rock to around $60 \mathrm{MPa}$ for highly competent rock (Goodman 1989; Bell 1999; Sivakugan et al. 2014). Based on these estimates, large-scale failure of the host rock around dykes is thus expected to be common.

In most of our models, we invoke the pore pressure of fluids in the host rock, and assume that this pore pressure is constant throughout the domain. The pore fluids may be volatiles that escape from the hot magma, they may be fluids (typically water) which existed in the host rock prior to dyke emplacement (possibly with pressure changes due to the heat from the magmatic intrusion), or they may be volatiles such as water or $\mathrm{CO}_{2}$ from solid phases (such as clays or carbonates) which are released because of heating of the host rock. By assuming a constant pore fluid pressure throughout, we implicitly neglect the process of fluid migration through the porous matrix. Clearly, this assumption is not generally met. Depending on the propagation velocity of the dyke and on the flow rate of the fluid (which in turn depends on viscosity, permeability and pressure and supply rate at the source), the fluid pressure is in general a function of space and time. Since the fluid pressure is typically highest near the dyke, it follows that the effective confining pressure, $p-p_{f}$, is lowest near the dyke, and increases with distance from the dyke. For fast-propagating dykes, and for dykes intruding into rocks with low permeability, the type of brittle failure (i.e. small-scale, moderatescale, or large-scale yielding) may thus be largely controlled by the interplay of dyke propagation and porous transport. Our results only show the two simple end-member cases, namely one without fluid effects (dyke propagates infinitely fast, or permeability is zero), and one with spatially constant fluid effects (porous transport is much faster than dyke propagation).

The concept of fracture toughness is used in many branches of Earth science, such as hydrofracturing (both on Earth and other terrestrial bodies, Thiercelin 1989; Desroches et al. 1994; Papanastasiou 1999; Neveu et al. 2015), propagation and stability of fractures and different magmatic intrusions (Rocchi et al. 2004; Rivalta \& Dahm 2004; Balme et al. 2004b; Rivalta \& Dahm 2006; Schultz et al. 2008; Jin \& Johnson 2008; Johnson \& Jin 2009; Schultz et al. 2010; Currier \& Marsh 2015; Kavanagh et al. 2017), blasting (Nath Singh \& Sun 1990; Hogan et al. 2016), rock shattering due to impacts (Ramesh et al. 2015; Tonge et al. 2016) and geoengineering (Rao et al. 2007). Clearly, a consistent and reliable measure of the resistance of different rock types to fracture propagation is needed, and the present state is unsatisfactory with different measurement techniques yielding fracture toughness values that vary by about two orders of magnitude. Our results indicate that the problem stems from fundamental differences in the process of fracturing in rock relative to most other materials, and as a result, fracture toughness in the traditional sense is often ill-defined. The overpressure in dykes is quite commonly of the same order as the yield strength of rock in shear. Under such conditions, the process zone at the dyke tip is of a spatial scale that is comparable to the dimensions of the dyke itself (e.g Figs 4a and b). The dyke-tip zone is thus described by large-scale plasticity, rather than small-scale plasticity, and hence any concepts and approximations based on LEFM are inaccurate.

Our results demonstrate the existence of two significantly different solutions to the problem of dyke propagation under large-scale 
yielding conditions, namely the IR model and the PR model. These models differ only in terms of the evolution history, but are otherwise identical (i.e. same boundary conditions and same material parameters). Any number of similar set-ups can be devised, for example by varying the fluid pressure during dyke formation, by incorporating the dynamics of magma flow, or by including effects of far-field stresses. Since the solutions for the stress field and for the total plastic deformation near the dyke are vastly different for the IR and PR models, it is clear that, under large-scale yielding, the near-tip solution does not decouple from the far-field solution in the same way as for small- and moderate-scale yielding. Likewise, models with different history or boundary conditions will generally differ in terms of the deformation solution in the vicinity of the dyke. This observation has an important effect on the strategy used for solving this type of fracture-mechanical problem. Whereas for small- and moderate-scale yielding, the stability of a fracture of given length can be obtained from consideration of the far-field stress solution alone (because the effect of the fracture has already been determined independently from the case of an isolated fracture), for large-scale yielding, the two problems are inherently coupled and must always be solved together. The two parameterizations for the IF and PR models (Table 1) are not only much more complicated to use than a the corresponding application of a single parameter such as the fracture toughness or the J-integral, but in addition, they are only valid for the specific cases described (i.e. formation at constant pressure in an infinite, unstressed medium). For similar cases, either the IR or the PR solution may be used as an approximation, but in general, the complete problem must be solved numerically.

Numerical models of fractures and dykes are generally limited by a finite resolution. An important question is whether the numerical models of large-scale plastic yielding need to be adjusted in order to properly model the physical processes that occur at the microscopic scale below the resolution limit. In some sense, incorporation of such processes at a microscopic scale is similar to the approach in models of small- and moderate-scale yielding, which use the purely elastic solution at the macroscopic scale while assuming plastic deformations to occur in a small zone near the fracture tip. However, there is an important difference. In the purely elastic LEFM model, the stresses become infinite at the fracture tip. A different mechanical process is thus required at the very minimum within a small region surrounding the tip, so that the stress field becomes bounded. At the same time, the elastic solution is known in analytical form, so there are no complications related to limited numerical resolution. On the other hand, the plastic solution in largescale yielding has a solution with all stress perturbations bounded by (or near) the plastic yield stress. If the assumed plastic yield function is valid everywhere and at all scales, the macroscopic (numerical) plastic model is thus sufficient for computing the deformation field as well as the energy terms of the Griffith energy balance throughout, including the region surrounding the fracture tip. In this case, more accurate results can always (at least in principle) be computed by increasing the spatial resolution, but the general character of the solution is not expected to change from that shown in Fig. 9.

Yet, even if different mechanical processes are not required for the large-scale yielding model, it is nonetheless possible that the physics is different at some small scale. For example, solidification of magma within the dyke tip (Bolchover \& Lister 1999) may increase the force required to extend the dyke. Alternatively, it is possible that the plastic yield stress varies as a function of spatial scale due to the size effect of material strength (Jaeger et al. 2009), which predicts greater strength on small spatial scales because of the lower probability that large-scale flaws exist in a relatively smaller volume. If these processes act on a spatial scale above the numerical resolution limit, they can be taken into account explicitly, such as by a local increase in yield stress at the dyke tip due to solidifying magma. Scale-dependence of plasticity due to the size effect can be described by non-local theories of plasticity (Bažant 1999; Voyiadjis \& Al-Rub 2005). On the other hand, when the microscopic scales are not explicitly resolved in the model (as is often the case in macroscopic fracture mechanics models), then the microscopic effects can be included in exactly the same way as for the small-scale yielding model of Orowan (1955) and Irwin (1957). We thus propose that the concept of fracture toughness is retained as a summary description of the small-scale processes at the fracture tip which are not modelled explicitly. Whether or not such a fracture toughness effect has a significant impact on the solution can only be determined experimentally. Given the much smaller volume associated with the microscopic process zone when compared with the volume simultaneously deforming in large-scale yielding, it is expected that the energies of the large-scale dissipative processes will typically strongly dominate whenever they are active.

The question then naturally arises whether the fracture toughness measured in the laboratory is the same as the small-scale fracture toughness due to microscopic processes in dyke settings. In laboratory experiments, toughness measurements using pressurized fractures use a fracture that is initiated by introducing a cut, and this cut is then jacketed in order to preclude any potential effects of the pore pressure (e.g. Clifton et al. 1976). Large- and moderate-scale failure is thus avoided, and the fracture toughness that is measured is appropriate for small-scale yielding conditions. However, the fracture tip conditions in the jacketed experiment are naturally dry, whereas the area immediately ahead of a natural dyke is generally expected to be affected by volatile pressure in the pore spaces. An additional difference between experiments and natural dykes is that the sample preparation for toughness measurements, which involves the cutting of a notch, is more similar to the IF model, whereas most dykes probably form by propagation and hydrofracturing (closer to the PR model). A third difference is that experiments are typically conducted on small, competent samples, while large rock masses contain relatively more larger cracks and joints (size effect). All three differences lead to enhanced non-elastic deformation in dyke settings, and thus in a greater apparent fracture toughness when only the microscopic scale is considered. While a conclusive answer is only possible based on more detailed laboratory studies, the above argument indicates that the fracture toughness measured in the laboratory is an underestimate of the small-scale fracture toughness in dykes (when only the mechanisms in the microscopic process zone are considered which corresponds to small-scale yielding). However, since the microscopic process zone is several orders of magnitude smaller (in volume) than the zone that deforms in large-scale yielding, its effect on the macroscopic energy balance is expected to be subordinate or even negligible.

\section{CONCLUSION}

The solid mechanical aspects of dyke propagation in host rock with different rheologies were analysed using an extended version of the Griffith energy balance, as this is the only method that retains its validity under large-scale yielding conditions. We mainly focus on elastoplasticity, which is used as a proxy for distributed shear fracturing and subsequent frictional sliding on the newly created fracture surfaces. Given the stress state at the dyke tip for typical 
magma overpressures of up to a few tens of MPa, we find that plastic failure is not limited to a small process zone as often assumed, but instead occurs over a large volume (so-called large-scale yielding) provided that either the lithostatic overburden is low (depths of up to a few $\mathrm{km}$ or tens of $\mathrm{km}$ ), or that the fluid pressure of water or volatiles in the host rock significantly aids in the process of fracturing and frictional sliding. Under these conditions, we find that

(1) The scale of plastic deformation depends mostly on the magma overpressure relative to the plastic yield stress. When the overpressure is about 1-2 times the yield stress, plastic failure occurs over a region that is comparable in size to the length of the dyke itself.

(2) Plasticity affects the energy balance by two mechanisms. The first mechanism is that of energy dissipation due to plastic flow. The second mechanism is characterized by additional dyke opening due to plastic deformation. Additional dyke opening causes an increase of the energy available for dyke propagation due to the pressure work against the moving dyke walls. The two plastic mechanisms thus work against each other.

(3) The final deformational state in incremental plasticity depends on the dynamic path taken to reach that state. We tested two models of geological interest. In the PR model, the dyke forms by propagation at constant pressure. In the IF model, the dyke forms by inflation of a pre-existing crack. The difference between a PR dyke and an IF dyke of equal length is that the PR dyke is surrounded by a broad halo of plastic deformation, while around the IF dyke, plastic deformation is limited to a zone surrounding the crack tip.

(4) In the PR model, dissipative processes dominate over the increase in boundary work due to plastic deformations. In this setup, plasticity causes a reduction in the the net energy available for fracturing, thus making the dyke more stable against propagation. In the IF model, the opposite is the case. The additional boundary work due to plasticity dominates over the total dissipation, making the dyke less stable against propagation.

(5) The effects of large-scale yielding depend on various parameters such as magma overpressure, plastic yield stress, dyke length, and the dynamic path to the final configuration. Due to these complications, it is impossible to summarize large-scale failure by a single parameter such as fracture toughness. Instead, the details of the large-scale failure model must be taken into account explicitly.

(6) Numerical macroscopic models of fractures explicitly compute stresses and displacements at the macroscale, which is determined by the resolution of the numerical grid. Processes which occur on spatial scales that are not resolved (typically about 0.001 or 0.0001 of the fracture length), which we term microscopic, can then be combined into a single parameter which plays essentially the same role as the fracture toughness in small-scale yielding. If this microscopic fracture toughness is due to the same plastic rheology as the macroscopic failure, then its contribution to the overall energy balance is negligible (because dissipation per unit volume is constant, and the microscopic process only operates over a negligible volume). The microscopic fracture toughness becomes significant only if there are very large forces acting on the microscale, such as fracture tip cohesion or the forces required to generate new free surfaces.

(7) Our results offer one possible explanation for the longstanding paradox that fracture toughnesses calculated from dyke observations are greater by a factor of about 100 relative to those derived from laboratory measurements. In laboratory measurements, the initial fracture is cut into the rock specimen (i.e. similar to the IF model), and effects of fluid pressure on shear failure are not present. The observed fracture toughness is therefore that of the elastic model under small-scale yielding assumptions (IF model with high yield stress). Most dykes probably form by propagation (PR model), and fluid pressure due to volatiles further enhances plastic deformation. The apparent fracture toughness (that is, the fracture toughness neglecting large-scale plastic deformations and incorrectly assuming small-scale yielding conditions) in the dyke calculations is of the observed magnitude if the magma overpressure is approximately two times greater than the actual yield stress.

\section{REFERENCES}

Abdelmalak, M.M., Mourgues, R., Galland, O. \& Bureau, D., 2012. Fracture mode analysis and related surface deformation during dyke intrusion: results from 2D experimental modelling, Earth. planet. Sci. Lett., 359360, 93-105.

Albino, F., Amelung, F. \& Gregg, P., 2018. The role of pore-fluid pressure on the failure of magma reservoirs: insights from Indonesian and Aleutian arc volcanoes, J. geophys. Res.: Solid Earth, p. 2017JB014523.

Anderson, T.L., 2017. Fracture Mechanics: Fundamentals and Applications, 4th edn, CRC Press.

Balme, M.R., Rocchi, V., Jones, C., Sammonds, P.R., Meredith, P.G. \& Boon, S., 2004a. Fracture toughness measurements on igneous rocks using a high-pressure, high-temperature rock fracture mechanics cell, $J$. Volc. Geotherm. Res., 132(2), 159-172.

Balme, M.R., Sammonds, P.R., Vita-Finzi, C. \& Couchman, J.P., 2004b. Experimental and theoretical fracture mechanics applied to fracture of the crust of Venus, J. geophys. Res. Planets, 109(E3), E03005.

Bažant, Z.P., 1999. Size effect on structural strength: a review, Arch. Appl. Mech., 69(9), 703-725.

Bell, F.G., 1999. Engineering Properties of Soil and Rocks, 4th edn, Blackwell Science.

Bolchover, P. \& Lister, J.R., 1999. The effect of solidification on fluid-driven fracture, with application to bladed dykes, Proc. R. Soc. Lond., A: Math., Phys. Eng. Sci., 455(1987), 2389-2409.

Clifton, R.J., Simonson, E.R., Jones, A.H. \& Green, S.J., 1976. Determination of the critical-stress-intensity factor KIc from internally pressurized thick-walled vessels, Exp. Mech., 16(6), 233-238.

Currier, R.M. \& Marsh, B.D., 2015. Mapping real time growth of experimental laccoliths: the effect of solidification on the mechanics of magmatic intrusion, J. Volc. Geotherm. Res., 302, 211-224.

Daniels, K.A., Kavanagh, J.L., Menand, T. \& Stephen, J.S.R., 2012. The shapes of dikes: evidence for the influence of cooling and inelastic deformation, Geol. soc. Am. Bull., 124(7-8), 1102-1112.

Delaney, P., Pollard, D., Ziony, J. \& Mckee, E., 1986. Field relations between dikes and joints - emplacement processes and paleostress analysis, J. geophys. Res.: Solid Earth Planets, 91(B5), 4920-4938.

Delaney, P.T. \& Pollard, D.D., 1981. Deformation of host rocks and flow of magma during growth of minette dikes and breccia-bearing intrusions near Ship Rock, New Mexico, US Geol. Surv. Prof. Pap. 1202.

Desroches, J., Detournay, E., Lenoach, B., Papanastasiou, P., Pearson, J. R.A., Thiercelin, M. \& Cheng, A., 1994. The crack tip region in hydraulic fracturing, Proc. R. Soc. Lond. A, 447(1929), 39-48.

Dyskin, A.V., 1997. Crack growth criteria incorporating non-singular stresses: size effect in apparent fracture toughness, Int. J. Fract., 83(2), 191-206.

Geshi, N., Kusumoto, S. \& Gudmundsson, A., 2010. Geometric difference between non-feeder and feeder dikes, Geology, 38(3), 195-198.

Goodman, R.E., 1989. Introduction to Rock Mechanics, Wiley.

Griffith, A.A., 1921. The phenomena of rupture and flow in solids, Phil. Trans. R. Soc. Lond. A, 221(582-593), 163-198.

Guldstrand, F., Burchardt, S., Hallot, E. \& Galland, O., 2017. Dynamics of surface deformation induced by dikes and cone sheets in a cohesive Coulomb brittle crust, J. geophys. Res.: Solid Earth, 122(10), 8511-8524.

Henyey, F.S. \& Pomphrey, N., 1982. Self-consistent elastic moduli of a cracked solid, Geophys. Res. Lett., 9(8), 903-906. 
Hertzberg, R.W., 2012. Deformation and Fracture Mechanics of Engineering Materials, 5th edn, John Wiley and Sons.

Hill, R., 1998. The Mathematical Theory of Plasticity, Vol. 11, Oxford University Press.

Hirth, G., Teyssier, C. \& Dunlap, J.W., 2001. An evaluation of quartzite flow laws based on comparisons between experimentally and naturally deformed rocks, Int. J. Earth Sci., 90(1), 77-87.

Hogan, J.D., Farbaniec, L., Daphalapurkar, N. \& Ramesh, K., 2016. On compressive brittle fragmentation, J. Am. Ceram. Soc., 99(6), 2159-2169.

Irwin, G., 1957. Analysis of stresses and strains near the end of a crack traversing a plate, J. Appl. Mech.

Jaeger, J.C., Cook, N.G. \& Zimmerman, R., 2009. Fundamentals of Rock Mechanics, John Wiley \& Sons.

Jin, Z.-H. \& Johnson, S.E., 2008. Magma-driven multiple dike propagation and fracture toughness of crustal rocks, J. geophys. Res., 113(B3), B03206.

Johnson, S.E. \& Jin, Z.-H., 2009. Magma extraction from the mantle wedge at convergent margins through dikes: a parametric sensitivity analysis, Geochem. Geophys. Geosyst., 10(8), Q08017.

Kavanagh, J. \& Sparks, R.S.J., 2011. Insights of dyke emplacement mechanics from detailed 3D dyke thickness datasets, J. Geol. Soc., 168(4), 965-978.

Kavanagh, J.L., Rogers, B.D., Boutelier, D. \& Cruden, A.R., 2017. Controls on sill and dyke-sill hybrid geometry and propagation in the crust: the role of fracture toughness, Tectonophysics, 698, 109-120.

Kusumoto, S., Geshi, N. \& Gudmundsson, A., 2013. Aspect ratios and magma overpressures of non-feeder dikes observed in the Miyake-jima volcano (Japan), and fracture toughness of its upper part, Geophys. Res. Lett., 40(6), 1065-1068.

Lawn, B.R. \& Wilshaw, T.R., 1975. Fracture of Brittle Solids, Cambridge University Press.

Lecampion, B., Bunger, A. \& Zhang, X., 2018. Numerical methods for hydraulic fracture propagation: a review of recent trends, J. Nat. Gas Sci. Eng., 49, 66-83.

Leopold, G. \& Munier, R., 2015. Crack propagation in large scale yielding (LSY) conditions, Proc. Eng., 133, 681-687.

Malvern, L.E., 1969. Introduction to the Mechanics of a Continuous Medium, Prentice-Hall.

Maurel, V. et al., 2017. Fatigue crack growth under large scale yielding condition: the need of a characteristic length scale, Int. J. Fatigue, 102, 184-201.

Nath Singh, R. \& Sun, G., 1990. Applications of fracture mechanics to some mining engineering problems, Min. Sci. Tech., 10(1), 53-60.

Neveu, M., Desch, S.J. \& Castillo-Rogez, J.C., 2015. Core cracking and hydrothermal circulation can profoundly affect Ceres' geophysical evolution, J. geophys. Res. Planets, 120(2), 123-154.

Orowan, E., 1955. Energy Criteria of Fracture, Vol. 34, Elsevier.

Papanastasiou, P., 1997. The influence of plasticity in hydraulic fracturing, Int. J. Fracture, 84(1), 61-79.

Papanastasiou, P., 1999. The effective fracture toughness in hydraulic fracturing, Int. J. Fract., 96(2), 127.

Papanastasiou, P. \& Thiercelin, M., 1993. Influence of inelastic rock behaviour in hydraulic fracturing, Int. J. Rock Mech. Min. Sci. Geomech. Abst., 30(7), 1241-1247.

Parfitt, E.A., 1991. The role of rift zone storage in controlling the site and timing of eruptions and intrusions of Kilauea Volcano, Hawaii, J. geophys. Res., 96(B6), $10101-10112$.

Paterson, M.S. \& Wong, T.-f., 2005. Experimental Rock Deformation: The Brittle Field, Springer Science \& Business Media.

Poland, M.P., Moats, W.P. \& Fink, J.H., 2008. A model for radial dike emplacement in composite cones based on observations from Summer Coon volcano, Colorado, USA, Bull. Volcanol., 70(7), 861-875.

Pollard, D.D., 1987. Elementary fracture mechanics applied to the structural interpretation of dykes, in Mafic Dyke Swarms, Vol. 34, pp. 5-24, eds Halls, H.C. \& Fahrig, W.F., Geol. Assoc. Can. Spec. Pap.

Pollard, D.D. \& Muller, O.H., 1976. The effect of gradients in regional stress and magma pressure on the form of sheet intrusions in cross section, $J$. geophys. Res., 81(5), 975-984.
Ramesh, K.T., Hogan, J.D., Kimberley, J. \& Stickle, A., 2015. A review of mechanisms and models for dynamic failure, strength, and fragmentation, Planet. Space Sci., 107, 10-23.

Ranalli, G., 1995. Rheology of the Earth, Springer Science \& Business Media.

Rao, Q.-h., Wang, Z., Xie, H.-f. \& Xie, Q., 2007. Experimental study of mechanical properties of sandstone at high temperature, J. Cent. South Univ. Technol., 14(1), 478-483.

Rice, J.R., 1968. A path independent integral and the approximate analysis of strain concentration by notches and cracks, J. appl. Mech., 35(2), 379386.

Rice, J.R., 1974. Limitations to the small scale yielding approximation for crack tip plasticity, J. Mech. and Phys. Solids, 22(1), 17-26.

Rivalta, E. \& Dahm, T., 2004. Dyke emplacement in fractured media: application to the 2000 intrusion at Izu islands, Japan, Geophys. J. Int., 157(1), 283-292.

Rivalta, E. \& Dahm, T., 2006. Acceleration of buoyancy-driven fractures and magmatic dikes beneath the free surface, Geophys. J. Int., 166(3), 1424-1439.

Rocchi, V., Sammonds, P.R. \& Kilburn, C. R.J., 2004. Fracturing of Etnean and Vesuvian rocks at high temperatures and low pressures, J. Volc. Geotherm. Res., 132(2), 137-157.

Rubin, A.M. \& Pollard, D.D., 1987. Origins of blade-like dikes in volcanic rift zones, U.S. Geol. Surv. Prof. Pap, p. 1350.

Scheibert, J., Galland, O. \& Hafver, A., 2017. Inelastic deformation during sill and laccolith emplacement: Insights from an analytic elastoplastic model, J. geophys. Res.: Solid Earth, 122(2), 923-945.

Schultz, R.A., Mège, D. \& Diot, H., 2008. Emplacement conditions of igneous dikes in Ethiopian Traps, J. Volc. Geotherm. Res., 178(4), 683692.

Schultz, R.A., Hauber, E., Kattenhorn, S.A., Okubo, C.H. \& Watters, T.R., 2010. Interpretation and analysis of planetary structures, J. Struct. Geol., 32(6), 855-875.

Sivakugan, N., Das, B.M., Lovisa, J. \& Patra, C.R., 2014. Determination of $\mathrm{c}$ and $\phi$ of rocks from indirect tensile strength and uniaxial compression tests, Int. J. Geotech. Eng., 8(1), 59-65.

Sneddon, I.N. \& Lowengrub, M., 1969. Crack Problems in the Classical Theory of Elasticity, Wiley.

Tada, H., Paris, P. \& Irwin, G., 2000. The Stress Analysis of Cracks Handbook, 3rd edn, McGraw-Hill.

Thiercelin, M., 1989. Fracture toughness and hydraulic fracturing, Int. J. Rock Mech. Min. Sci. Geomech. Abstr., 26(3), 177-183.

Timoshenko, S. \& Goodier, J., 1970. Theory of Elasticity, 3rd edn, McGrawHill.

Tonge, A.L., Ramesh, K.T. \& Barnouin, O., 2016. A model for impactinduced lineament formation and porosity growth on Eros, Icarus, 266, 76-87.

Townsend, M., Pollard, D.D., Johnson, K. \& Culha, C., 2015. Jointing around magmatic dikes as a precursor to the development of volcanic plugs, Bull. Volcanol., 77(10), 92.

Unger, D.J., 2001. Analytical Fracture Mechanics, Courier Corporation.

Vachon, R. \& Hieronymus, C.F., 2017. Effect of host-rock rheology on dyke shape, thickness and magma overpressure, Geophys. J. Int., 208(3), 1414-1429.

Voyiadjis, G.Z. \& Al-Rub, R.K.A., 2005. Gradient plasticity theory with a variable length scale parameter, Int. J. Solids Struct., 42(14), 3998-4029.

Walsh, J., 1965. The effect of cracks on the compressibility of rock, $J$. geophys. Res., 70(2), 381-389.

Weinberger, R., Lyakhovsky, V., Baer, G. \& Agnon, A., 2000. Damage zones around en echelon dike segments in porous sandstone, J. geophys. Res.: Solid Earth, 105(B2), 3115-3133

Westergaard, H.M., 1933. Stresses at a crack, size of the crack, and the bending of reinforced concrete, J. Proc., 30(11), 93-102.

Yu, M.-H., Ma, G.-W., Qiang, H.-F. \& Zhang, Y.-Q., 2006. Generalized Plasticity, Springer Science \& Business Media.

Zhang, Q.B. \& Zhao, J., 2014. Quasi-static and dynamic fracture behaviour of rock materials: phenomena and mechanisms, Int. J. Fract., 189(1), $1-32$. 


\section{SUPPORTING INFORMATION}

Supplementary data are available at $G J I$ online.

Figure S1. Propagation forces for a dyke in elastoplastic host rock with Drucker-Prager failure criterion as functions of overburden pressure $p_{o b}$. Model uses $P R$ set-up, and parameters are $a=$ $10000 \mathrm{~m}, C=15 \mathrm{MPa}, \alpha=0.25$. The fluid pressure in the failure envelope is set to $p_{f}=0$. The model approaches the elastic solution at high $p_{o b}$, but even at $p_{o b}=100 \mathrm{MPa}$ (around $40 \mathrm{~km}$ depth) plastic effects are significant.

Figure S2. Propagation forces for a dyke in elastoplastic host rock with Drucker-Prager failure criterion in the model set-up $P R$. Functional dependence is on the fluid pressure $p_{f}$ in the Drucker-Prager law, which is set equal to the magma overpressure, that is $p_{f}=\Delta p$. Parameters are $a=10000 \mathrm{~m}, C=15 \mathrm{MPa}, \alpha=15 \mathrm{MPa}$. Results are similar to those of the corresponding plastic model with constant yield stress, showing that the dynamic pressure in the DruckerPrager law has only a minor effect on the solution.

Figure S3. Propagation forces $F$ as functions of half-length $a$ for the pressure-dependent elastic model with $\Delta p=10 \mathrm{MPa}$. The functional dependence of the elastic moduli $K(p)$ and $G(p)$ is given by the empirical fit of Vachon \& Hieronymus (2017). Dashed lines represent the best linear fit to the model data (stars and dots). $F_{e}$ and $F_{\text {tot }}$ are plot on top of each other. All $F$-terms are higher by a factor of about 1.3 relative to the linear elastic model.

Please note: Oxford University Press is not responsible for the content or functionality of any supporting materials supplied by the authors. Any queries (other than missing material) should be directed to the corresponding author for the paper. 